\title{
Some Remarks on Avalanches Modelling: An Introduction to Shallow Flows Models
}

\author{
Enrique D. Fernández-Nieto and Paul Vigneaux
}

These notes are dedicated to D. Antonio Valle Sánchez (1930-2012). D. Antonio was the first Spanish PhD student of Jacques-Louis Lions. He can be considered as one of the founders of modern Applied Mathematics in Spain.

\begin{abstract}
The main goal of these notes is to present several depth-averaged models with application in granular avalanches. We begin by recalling the classical Saint-Venant or Shallow Water equations and present some extensions like the Saint-Venant-Exner model for bedload sediment transport. The first part is devoted to the derivation of several avalanche models of Savage-Hutter type, using a depth-averaging procedure of the 3D momentum and mass equations. First, the Savage-Hutter model for aerial avalanches is presented. Two other models for partially fluidized avalanches are then described: one in which the velocities of both the fluid and the solid phases are assumed to be equal, and another one in which both velocities are unknowns of the system. Finally, a Savage-Hutter model for submarine avalanches is derived. The second part is devoted to non-newtonian models, namely viscoplastic fluids. Indeed, a one-phase viscoplastic model can also be used to simulate fluidized avalanches. A brief introduction to Rheology and plasticity is presented in order to explain the Herschel-Bulkley constitutive law. We finally present the derivation of a shallow Herschel-Bulkley model.
\end{abstract}

E.D. Fernández-Nieto $(\bowtie)$

Dpto. Matemática Aplicada I, Universidad de Sevilla, Sevilla, Spain

e-mail: edofer@us.es

P. Vigneaux

Unité de Mathématiques Pures et Appliquées, Ecole Normale Supérieure de Lyon, Lyon, France

e-mail: Paul.Vigneaux@math.cnrs.fr 


\section{Introduction: Shallow Water Equations}

The classical shallow water equations were first derived in 1871 by Saint-Venant (see [46]). This system of equations describe the motion of a shallow layer of fluid in a channel, a lake, coastal areas, etc.

Several extensions of these classical equations have been proposed in the literature. For example, in [25] Gerbeau and Perthame propose a viscous Shallow Water model. They perform the asymptotic analysis of the Navier-Stokes equations where friction effects at the bottom have been taken into account. While in a first order approximation the viscous terms do not appear in the equations, a second order is needed to get them. In [35], a viscous one layer 2D Shallow-Water system is derived, by including a surface-tension term associated to the capillary effects at the free surface and a quadratic friction term at the bottom. These terms have been useful to prove the existence of global weak solutions in [13].

In the simple case of a rectangular channel with constant width and a fixed bottom topography (see Fig. 1), the Shallow Water equations are

$$
\left\{\begin{array}{l}
\partial_{t} h+\partial_{x}(h u)=0, \\
\partial_{t}(h u)+\partial_{x}\left(h u^{2}+\frac{1}{2} g h^{2}\right)=-g h \partial_{x} z_{b}-g h S_{f},
\end{array}\right.
$$

where $x$ denotes the horizontal variable through the axis of the channel and $t$ is the time variable. $u(x, t)$ and $h(x, t)$ represent the velocity and the height of the water column, respectively. $g$ is the gravity and $z_{b}(x)$ the bottom topography (see Fig. 1).

The term $S_{f}$ models the friction forces. In the particular case of the Manning law we have

$$
S_{f}=\frac{g \eta^{2}|u| u}{R_{h}^{4 / 3}},
$$

where $\eta$ is the Manning's coefficient and $R_{h}$ is the hydraulic radius, which can be approximated by $h$.

The Saint-Venant-Exner equations take into account the bed-load sediment transport. In this case, we have the Shallow Water or Saint-Venant system coupled with a continuity equation to model the evolution of the sediment layer,

$$
\left\{\begin{array}{l}
\partial_{t} h+\partial_{x}(h u)=0 \\
\partial_{t}(h u)+\partial_{x}\left(h u^{2}+\frac{1}{2} g h^{2}\right)=-g h \partial_{x} z_{b}-g h S_{f}, \\
\partial_{t} z_{b}+\xi \partial_{x} q_{b}=0
\end{array}\right.
$$

where $\xi=1 /\left(1-\psi_{0}\right)$ and $\psi_{0}$ is the porosity of the sediment layer. $q_{b}=$ $q_{b}(h, q)$ represents the solid transport discharge. The definition of the solid transport 
Fig. 1 Shallow water equations with a fixed bottom

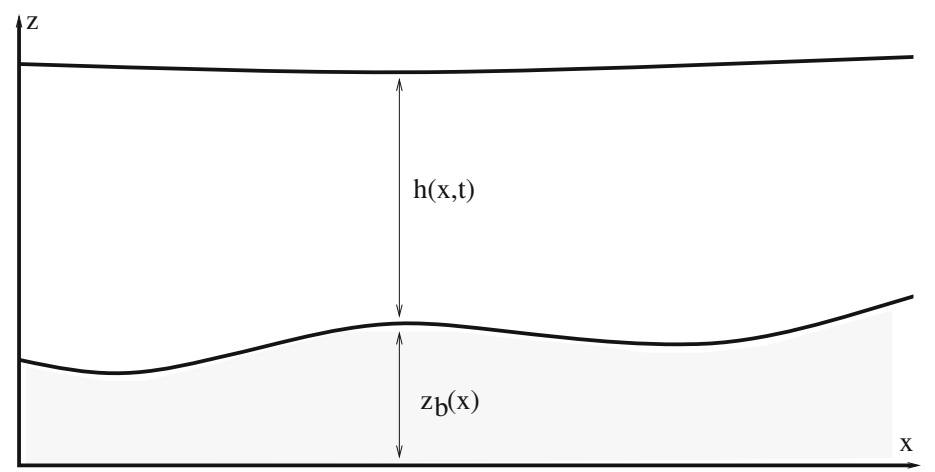

discharge is set usually by empirical laws. In the Appendix some classical formulae are presented.

Far to be exhaustive, several extensions of the Shallow Water equations can be mentioned: models that take into account varying bottom topography $[11,18,24]$; models to study erosion phenomena with local coordinate variable in space and time [12] or to study flows in rotating drums [27]; models that take into account dispersive effects [33]; models for two-layer stratified flows with viscosity and capillarity [39], turbidity currents models [38], multilayer shallow water models to incorporate tridimensional effects $[7,23] \ldots$

In the pioneering work of Savage and Hutter [47], a shallow-water type model has been proposed to study aerial avalanches. In the following section we describe the derivation of the Savage-Hutter model. The classical Shallow Water system is the particular case of the Savage-Hutter model obtained by neglecting the Coulomb friction term. Therefore, its derivation from Navier-Stokes equations is a particular case of the general study presented in next section.

In the following sections, the derivation of several shallow water type models to study three different types of avalanches are presented. Sections 2-6 correspond to Savage-Hutter type models for aerial avalanches, partially fluidized aerial avalanches and submarine avalanches (see [21]). In Sect.7, we present a brief introduction to Rheology and plasticity in order to explain the constitutive equation of the Herschel-Bulkley model. A depth-averaged Herschel-Bulkley model is presented in Sect. 8. This model is a one-phase approach to study solid-fluid mixtures avalanches and represents an alternative to the two-phase Coulomb approach.

\section{Savage-Hutter Model for Aerial Avalanches}

Numerical modelling of sub-aerial debris or snow avalanches has been extensively investigated during this last decade with application to both laboratory experiments dealing with granular flows and geological events (see for example $[2,5,6,12,27$, $31,34,50])$. Most of the models devoted to gravitational granular flows describe the behavior of dry granular material following the pioneering work of Savage and Hutter (see [47]) in which a shallow water type model (i.e. thin layer approximation 
Fig. 2 Local coordinates

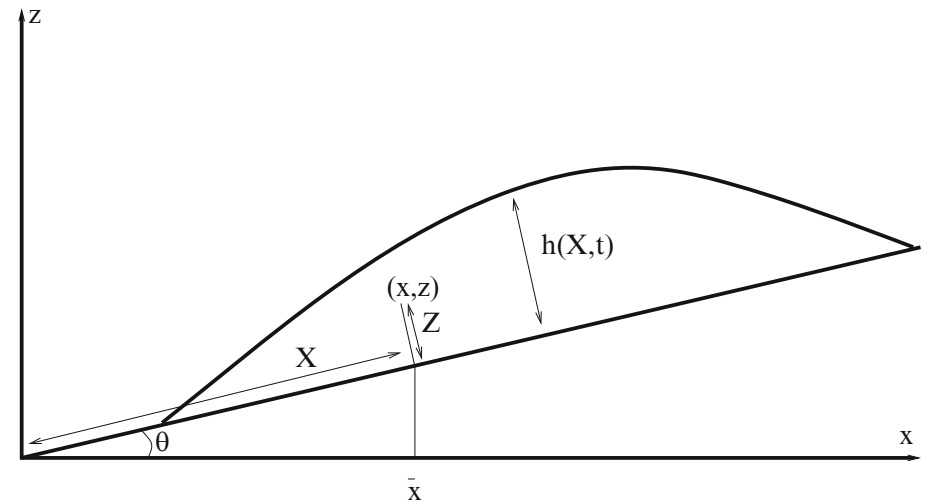

for a continuum medium) is derived to describe granular flows over a slopping plane based on Mohr-Coulomb considerations: a Coulomb friction is assumed to reflect the avalanche/bottom interaction and the normal stress tensor is defined by a constitutive law relating the longitudinal and the normal stresses through a proportionality factor $K$.

New Savage-Hutter models over a general bottom have been proposed. For example in [11], Bouchut et al., propose a Savage-Hutter type model for aerial avalanche which takes into account the curvature of the bottom. A two-layer Shallow Water type model with compressible effects has been introduced in [37] by Morales de Luna. He considers an upper compressible layer and a lower incompressible layer.

In this section, we present the derivation of the Savage-Hutter model over a plane with constant slope. First, we consider the Euler equations in Cartesian coordinates $\mathbf{X}=(x, z)$,

$$
\begin{gathered}
\mathbf{V}=\left(\begin{array}{c}
u \\
w
\end{array}\right), \quad \nabla \cdot \mathbf{V}=0, \\
\partial_{t}(\rho \mathbf{V})+\rho \mathbf{V} \cdot \nabla_{\mathbf{X}} \mathbf{V}=-\nabla \cdot P+\rho \nabla_{\mathbf{X}}(\mathbf{g} \cdot \mathbf{X}),
\end{gathered}
$$

where $\mathbf{g}=(0,-g), g$ being the gravity acceleration, $\mathbf{V}$ is the velocity field and $\rho$, the density of the granular layer. Moreover, we denote by $P$ the negative Cauchy stress tensor, also named pressure tensor,

$$
P=\left(\begin{array}{ll}
p_{x} & p_{x z} \\
p_{z x} & p_{z z}
\end{array}\right),
$$

with $p_{x z}=p_{z x}$.

Let us rewrite first the Euler equations in local coordinates $(X, Z)$ on an inclined plain whose slope is $\tan (\theta)$ (see Fig. 2). $Z$ is the distance between the points $(x, z)$ and $(\bar{x}, b(\bar{x}))$, where

$$
b(\bar{x})=\tan (\theta) \bar{x} .
$$


That is, $Z$ is the distance to the bed, measured along the normal direction and $X$ measures the arc length along the inclined plain. $\bar{x}$ is the $\mathrm{x}$-Cartesian coordinate of the point $(X, 0)$ (see Fig. 2$)$.

Let $h(x, t)$ be the height of the granular layer along the normal direction to the bed. The domain is

$$
\{(X, Z) ; \quad X \in[0, L], 0<Z<h(X, t)\} .
$$

The relation between the Cartesian coordinates $\mathbf{X}=(x, z)$ and the coordinates $(\bar{x}, Z)$ is

$$
\mathbf{X}=(\bar{x}-Z \sin \theta, b(\bar{x})+Z \cos \theta))
$$

where $(\bar{x}, b(\bar{x}))$ is a point of the bed.

The following definitions will also be used:

- $U$ and $W$ are the tangential and normal velocities, respectively,

$$
\left(\begin{array}{c}
U \\
W
\end{array}\right)=\left(\begin{array}{cc}
\cos \theta & \sin \theta \\
-\sin \theta & \cos \theta
\end{array}\right) \mathbf{V}
$$

- And $\mathscr{P}$ is the rotated Cauchy stress tensor:

$$
\mathscr{P}=\left(\begin{array}{cc}
\cos \theta & \sin \theta \\
-\sin \theta & \cos \theta
\end{array}\right) P\left(\begin{array}{cc}
\cos \theta & -\sin \theta \\
\sin \theta & \cos \theta
\end{array}\right)=\left(\begin{array}{ll}
\mathscr{P}_{X X} & \mathscr{P}_{X Z} \\
\mathscr{P}_{\mathrm{ZX}} & \mathscr{P}_{\mathrm{ZZ}}
\end{array}\right)
$$

Note that, as $p_{x z}=p_{x z}$, then $\mathscr{P}_{X Z}=\mathscr{P}_{Z X}$.

Equations (4) and (5) are re-written in the new variables as follows:

$$
\left\{\begin{array}{l}
\partial_{X}(U)+\partial_{Z}(W)=0, \\
\rho \partial_{t}(U)+\rho \partial_{X}\left(U^{2}\right)+\rho \partial_{Z}(W U)-\rho \partial_{X}(\mathbf{g} \cdot \mathbf{X})=-\partial_{X}\left(\mathscr{P}_{X X}\right)-\partial_{Z}\left(\mathscr{P}_{X Z}\right), \\
\rho \partial_{t}(W)+\rho \partial_{X}(U W)+\rho \partial_{Z}\left(W^{2}\right)-\rho \partial_{Z}(\mathbf{g} \cdot \mathbf{X})=-\partial_{X}\left(\mathscr{P}_{Z X}\right)-\partial_{Z}\left(\mathscr{P}_{Z Z}\right) .
\end{array}\right.
$$

In what follows, the derivation of the model proposed by Savage and Hutter in [47] to study aerial avalanches is described following the items:

- $[\partial]$ Boundary and kinematic conditions.

- $[\tilde{A}]$ Dimensional analysis.

- $[\uparrow]$ Hydrostatic pressure and constitutive law.

- $[M]$ Momentum conservation law. 
- $\left[\int\right]$ Integration process.

- $[\hookrightarrow]$ Final system of equations.

\section{1 [j] Boundary and Kinematic Conditions}

We denote by $\mathbf{n}^{h}$ the unit normal vector to the free granular surface $Z=h$ with positive vertical component, and by $\mathbf{n}^{0}=(0,1)$ the unit normal vector to the bottom $(Z=0)$.

The following kinematic condition is considered

$$
\partial_{t} h+\left.U\right|_{Z=h} \partial_{X} h-\left.W\right|_{Z=h}=0,
$$

which means that the particles at the free surface are transported with velocity $\left(\left.U\right|_{Z=h},\left.W\right|_{Z=h}\right)$.

The following boundary conditions are imposed:

- On $Z=h$ :

$$
\begin{gathered}
\mathbf{n}^{h} \cdot \mathscr{P} \mathbf{n}^{h}=0 \\
\mathscr{P} \mathbf{n}^{h}-\mathbf{n}^{h}\left(\mathbf{n}^{h} \cdot \mathscr{P} \mathbf{n}^{h}\right)=\left(\begin{array}{c}
\operatorname{fric}_{h}(U) \\
0
\end{array}\right) \quad i=1,2,
\end{gathered}
$$

where $\operatorname{fric}_{h}(U)$ is the friction term between the granular layer and the air. For the sake of simplicity we will suppose that $\operatorname{rric}_{h}(U)=0$.

- On $Z=0$ :

$$
\begin{gathered}
(U, W) \cdot \mathbf{n}^{0}=0 \quad \Rightarrow \quad W=0, \\
\mathscr{P} \mathbf{n}^{0}-\mathbf{n}^{0}\left(\mathbf{n}^{0} \cdot \mathscr{P} \mathbf{n}^{0}\right)=\left(\begin{array}{c}
-\left.\mathbf{n}^{0} \cdot \mathscr{P} \mathbf{n}^{0} \frac{U}{|U|}\right|_{Z=0} \tan \delta_{0} \\
0
\end{array}\right) .
\end{gathered}
$$

This last condition corresponds to a Coulomb friction law, defined in terms of the angle of repose $\delta_{0}$ (see [47]).

\section{$2.2[\tilde{A}]$ Dimensional Analysis}

Next, a dimensional analysis of the set of Eqs. (8), the kinematic and boundary conditions is performed. The non-dimensional variables $(\tilde{.})$ read: 


$$
\begin{aligned}
& (X, Z, t)=\left(L \tilde{X}, H \tilde{Z},(L / g)^{1 / 2} \tilde{t}\right), \\
& (U, W)=(L g)^{1 / 2}(\tilde{U}, \varepsilon \tilde{W}) \\
& h=H \tilde{h} \\
& \left(\mathscr{P}_{X X}, \mathscr{P}_{Z Z}\right)=g H\left(\tilde{\mathscr{P}}_{X X}, \tilde{\mathscr{P}}_{Z Z}\right), \\
& \mathscr{P}_{X Z}=g H \mu \tilde{\mathscr{P}}_{X Z},
\end{aligned}
$$

where:

- $\mu=\tan \delta_{0}, \delta_{0}$ being the angle of repose in the Coulomb term.

- By $L$ and $H$, we denote, respectively, the tangential and normal characteristic lengths.

- $\varepsilon=H / L$, which is supposed to be small: the Savage-Hutter model has been shown to reproduce experimental granular collapse over horizontal plane for aspect ratio $\epsilon \leq 0.5$, see [34].

Using the above change of variables, the system of Eqs. (8) is re-written as follows:

$$
\begin{gathered}
\partial_{X}(U)+\partial_{Z}(W)=0 \\
\partial_{t}(\rho U)+\rho U \partial_{X} U+\rho W \partial_{Z} U+\rho \partial_{X}\left(b+Z \cos \theta+\frac{\mathscr{P}_{X X}}{\rho}\right) \varepsilon=-\mu \partial_{Z}\left(\mathscr{P}_{X Z}\right) \\
\varepsilon\left\{\partial_{t}(\rho W)+\rho U \partial_{X}(W)+\rho W \partial_{Z}(W)+\partial_{X}\left(\mathscr{P}_{X Z}\right)\right\}+ \\
+\rho \partial_{Z}(b+Z \cos \theta)=-\partial_{Z}\left(\mathscr{P}_{Z Z}\right)
\end{gathered}
$$

where tildes have been dropped for simplicity.

The kinematic condition (9) is re-written as:

$$
\partial_{t} h+\left.U\right|_{Z=h} \partial_{X} h-\left.W\right|_{Z=h}=0 .
$$

Finally, the boundary conditions (10)-(13) are now given by:

- On $Z=h$, we have $\mathbf{n}^{h}=\left(-\varepsilon \partial_{X} h, 1\right) / \varphi^{S}$ with $\varphi^{S}=\sqrt{1+\varepsilon^{2}\left(\partial_{X} h\right)^{2}}$, then from (10) and (11) we obtain

$$
\begin{aligned}
& -\varepsilon \partial_{X} h \mathscr{P}_{X X}+\mu \mathscr{P}_{Z X}=0, \\
& -\varepsilon \partial_{X} h \mu \mathscr{P}_{X Z}+\mathscr{P}_{Z Z}=0 .
\end{aligned}
$$


- On $Z=0$, we have $\mathbf{n}^{0}=(0,1)$, then from (12) and (13) we obtain

$$
\begin{gathered}
\left.W\right|_{Z=0}=0 \\
\mu \mathscr{P}_{X Z}=-\left.\mathscr{P}_{Z Z} \frac{U}{|U|}\right|_{Z=0} \tan \delta_{0} .
\end{gathered}
$$

\section{$2.3[1]$ Hydrostatic Pressure and Constitutive Law}

From (17) we obtain

$$
\partial_{Z}\left(\mathscr{P}_{Z Z}\right)=-\rho \cos \theta+\mathscr{O}(\varepsilon)
$$

If we integrate (23) from $Z>0$ to $h$, we have, up to order $\varepsilon$,

$$
\mathscr{P}_{\mathrm{ZZ}}=\rho(h-Z) \cos \theta \text {. }
$$

The following constitutive law is considered (see [47])

$$
\mathscr{P}_{\mathrm{XX}}=K \mathscr{P}_{\mathrm{ZZ}}
$$

where $K$ measures the anisotropy or normal stress effects: while $K=1$ corresponds to isotropic conditions, $K \neq 1$ makes 'overburden pressures' different from the normal stresses parallel to the basal surface. In the case of the Shallow Water equations, $K=1$ is assumed.

The coefficient $K$ is defined according to the motion of the granular layer (see [45]):

$$
K=\left\{\begin{array}{l}
K_{a c t} \text { if } \partial_{X} U>0 \\
K_{\text {pas }} \text { if } \partial_{X} U<0
\end{array}\right.
$$

with

$$
K_{\text {act } / p a s}=2 \sec ^{2} \phi\left(1 \mp\left(1-\cos ^{2} \phi \sec ^{2} \delta_{0}\right)^{1 / 2}\right)-1
$$

being $\phi$ the internal friction angle, defined in terms of the type of grains and size.

The definition of $K$ can be done in different ways. For example, while in [28] Heinrich et al. consider $K=1$, other definitions of $K$ can be found in [29]. The effects related to the definition of $K$ in numerical modelling of experimental and natural flows is studied in $[43,45]$.

Using the previous relations, we have, up to order $\epsilon$,

$$
\mathscr{P}_{X X}=K \mathscr{P}_{\mathrm{ZZ}}=K \rho(h-Z) \cos \theta \text {. }
$$




\section{$2.4[M]$ Momentum Conservation Law: With Hydrostatic Pressure and Anisotropy of the Normal Stress}

By replacing (24) and (25) in (16) and using the incompressibility equation (15), we obtain, up to second order,

$\partial_{t}(\rho U)+\rho \partial_{X} U^{2}+\rho \partial_{Z}(U W)+\rho \partial_{X}(b+Z \cos \theta+K(h-Z) \cos \theta) \varepsilon=-\mu \partial_{Z}\left(\mathscr{P}_{X Z}\right)$

\section{$2.5\left[\int\right]$ Integration Process}

In this section, the mass equation (15) and the momentum equation (26) are depthaveraged in the normal direction. Let us introduce the following notation: we denote by $\bar{U}$ the average of the velocity along the normal direction:

$$
\bar{U}=\frac{1}{h} \int_{0}^{h} U(X, Z) d Z .
$$

We also introduce the notation:

$$
\overline{U^{2}}=\frac{1}{h} \int_{0}^{h} U^{2}(X, Z) d Z .
$$

If Eq. (15) is integrated from $Z=0$ to $Z=h$, we obtain

$$
0=\partial_{X}(h \bar{U})-\left.U\right|_{Z=h} \partial_{X} h+\left.W\right|_{Z=h}-\left.W\right|_{Z=0} .
$$

Now, using (18) and (21), the averaged mass equation is obtained

$$
\partial_{t} h+\partial_{X}(h \bar{U})=0 .
$$

Let us now integrate Eq. (26) from $Z=0$ to $Z=h$. As in the previous case, we use the kinematic condition (18) to obtain

$$
\begin{aligned}
\partial_{t}(h \bar{U})+\partial_{X}\left(h \overline{U^{2}}\right)+\left(\int_{0}^{h} \partial_{X}(b+Z \cos \theta+K(h-Z) \cos \theta) d Z\right) \varepsilon \\
\quad=-\frac{\mu}{\rho}\left(\mathscr{P}_{X Z}(h)-\mathscr{P}_{X Z}(0)\right) .
\end{aligned}
$$


Moreover, we have

$$
\int_{0}^{h} \partial_{X}(b+Z \cos \theta+(h-Z) \cos \theta K) d Z=h \partial_{X} b+\partial_{X}\left(\frac{h^{2}}{2} \cos \theta K\right) .
$$

By replacing this last expression in (27) we obtain the equation

$$
\partial_{t}(h \bar{U})+\partial_{X}\left(h \overline{U^{2}}+\varepsilon \frac{h^{2}}{2} \cos \theta K\right)=-\varepsilon h \partial_{X} b-\frac{\mu}{\rho}\left(\mathscr{P}_{X Z}(h)-\mathscr{P}_{X Z}(0)\right) .
$$

Then, the boundary conditions and the constitutive laws are used to derive $\mu \mathscr{P}_{X Z}(h)$ and $\mu \mathscr{P}_{X Z}(0)$ :

- From (19), by using (24) and $\mathscr{P}_{X X}=K \mathscr{P}_{\text {ZZ }}$, we have

$$
\mu \mathscr{P}_{X Z}(h)=\varepsilon \partial_{X} h \mathscr{P}_{\mathrm{ZZ}} K
$$

In [27] Gray introduced the assumption that the Coulomb term is of order $\gamma$ for some $\gamma \in(0,1)$. That is, $\mu=\tan \delta_{0}=\mathscr{O}\left(\varepsilon^{\gamma}\right)$. Under this assumption, we have

$$
\mu \mathscr{P}_{X Z}(h)=\mathscr{O}\left(\varepsilon^{1+\gamma}\right) .
$$

- Using Eq. (22), we obtain

$$
\frac{\mu}{\rho} \mathscr{P}_{X Z}(0)=-\left.\frac{\mathscr{P}_{Z Z}(0)}{\rho} \frac{U}{|U|}\right|_{Z=0} \tan \delta_{0}=-\left.h \cos \theta \frac{U}{|U|}\right|_{Z=0} \tan \delta_{0} .
$$

Therefore, assuming $\tan \delta_{0}=\mathscr{O}\left(\varepsilon^{\gamma}\right)$, we have

$$
\frac{\mu}{\rho} \mathscr{P}_{X Z}(0)=-\left.h \cos \theta \frac{U}{|U|}\right|_{Z=0} \tan \delta_{0}+\mathscr{O}\left(\varepsilon^{1+\gamma}\right) .
$$

Finally, substituting (29) and (30) in (28), the averaged momentum equation is obtained:

$\partial_{t}(h \bar{U})+\partial_{X}\left(h \overline{U^{2}}+\varepsilon \frac{h^{2}}{2} \cos \theta K\right)=-\varepsilon h \partial_{X} b-\left.h \cos \theta \frac{U}{|U|}\right|_{Z=0} \tan \delta_{0}+\mathscr{O}\left(\varepsilon^{1+\gamma}\right)$.

\section{$2.6[\hookrightarrow]$ Final System of Equations}

Coming back to the original variables, using (14), neglecting terms of order $\varepsilon^{1+\gamma}$ and supposing a constant profile of the velocities, the following system is obtained: 


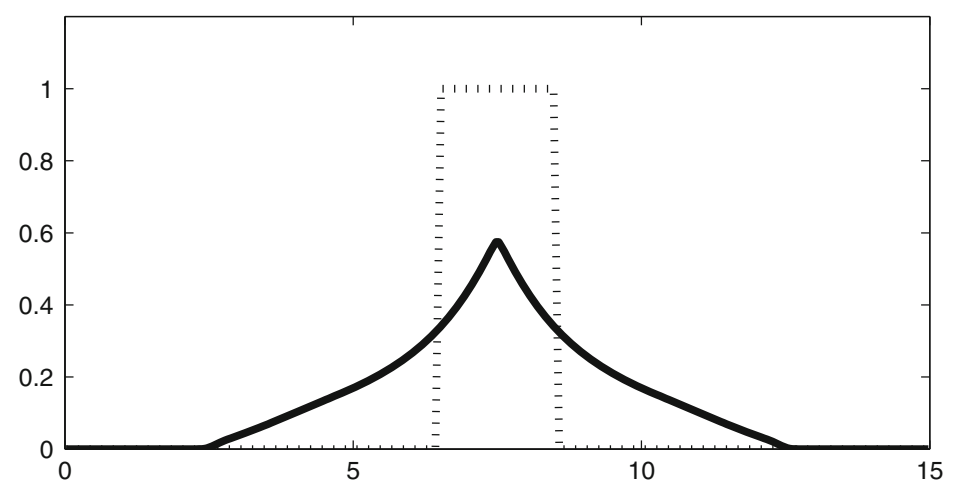

Fig. 3 Savage-Hutter model. Dotted line: initial profile of the granular layer. Continuous line: stationary profile of the granular layer

$$
\left\{\begin{array}{l}
\partial_{t} h+\partial_{X}(h \bar{U})=0 \\
\partial_{t}(h \bar{U})+\partial_{X}\left(h \bar{U}^{2}+g \cos \theta \frac{h^{2}}{2} K\right)=-g h \partial_{X} b+\mathscr{T},
\end{array}\right.
$$

where $\mathscr{T}$ represents the Coulomb friction term. This term must be understood as follows:

$$
\begin{aligned}
& \text { If }|\mathscr{T}| \geq \sigma_{c} \quad \Rightarrow \quad \mathscr{T}=-g h \cos \theta \frac{\bar{U}}{|\bar{U}|} \tan \delta_{0}, \\
& \text { If }|\mathscr{T}|<\sigma_{c} \quad \Rightarrow \quad \bar{U}=0,
\end{aligned}
$$

where $\sigma_{c}=g h \cos \theta \tan \delta_{0}$.

Let us illustrate the effects of the Coulomb friction term. We consider a test case consisting of a granular layer over a flat bottom whose initial profile is rectangular. The evolution of the layer is simulated by numerically solving System (31). Let us stress the importance of an adequate treatment of the Coulomb friction term (32) to obtain satisfactory numerical results (see for example [34]). In Fig. 3 the continuous line corresponds to the stationary profile of the granular layer for $\delta_{0}=25^{\circ}$. The initial condition is represented too (dotted line). The main difference between the classical Shallow Water equations and the Savage-Hutter model is the presence of the Coulomb friction term: if a closed domain is considered and the Coulomb friction term is neglected, the stationary solution is a horizontal free surface, corresponding to water at rest.

In Fig. 4 the evolution of the granular layer surface and its discharge is represented at several times. Observe that, while at $t=2$ the solution is stationary, at $t=1.5$ only the front of the avalanche is still moving. 

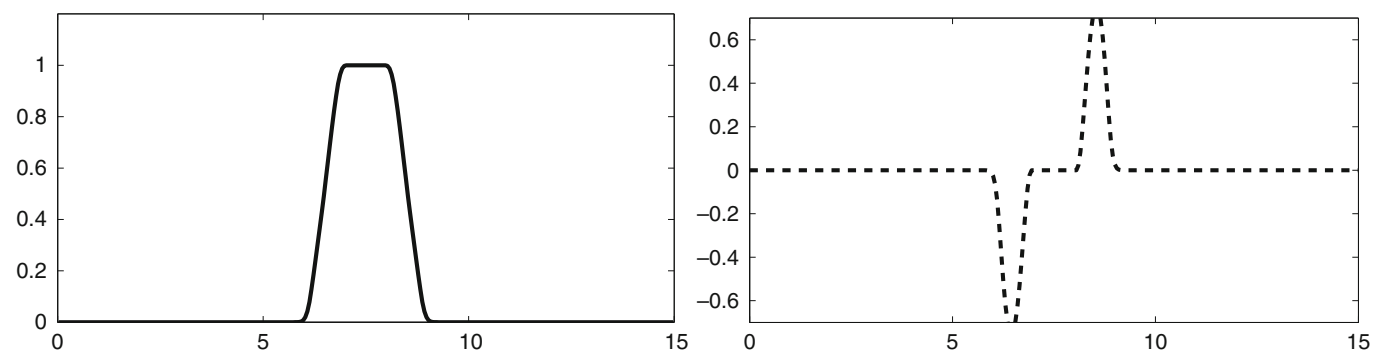

h $\quad \mathrm{t}=0.1 \mathrm{~s}$.

q
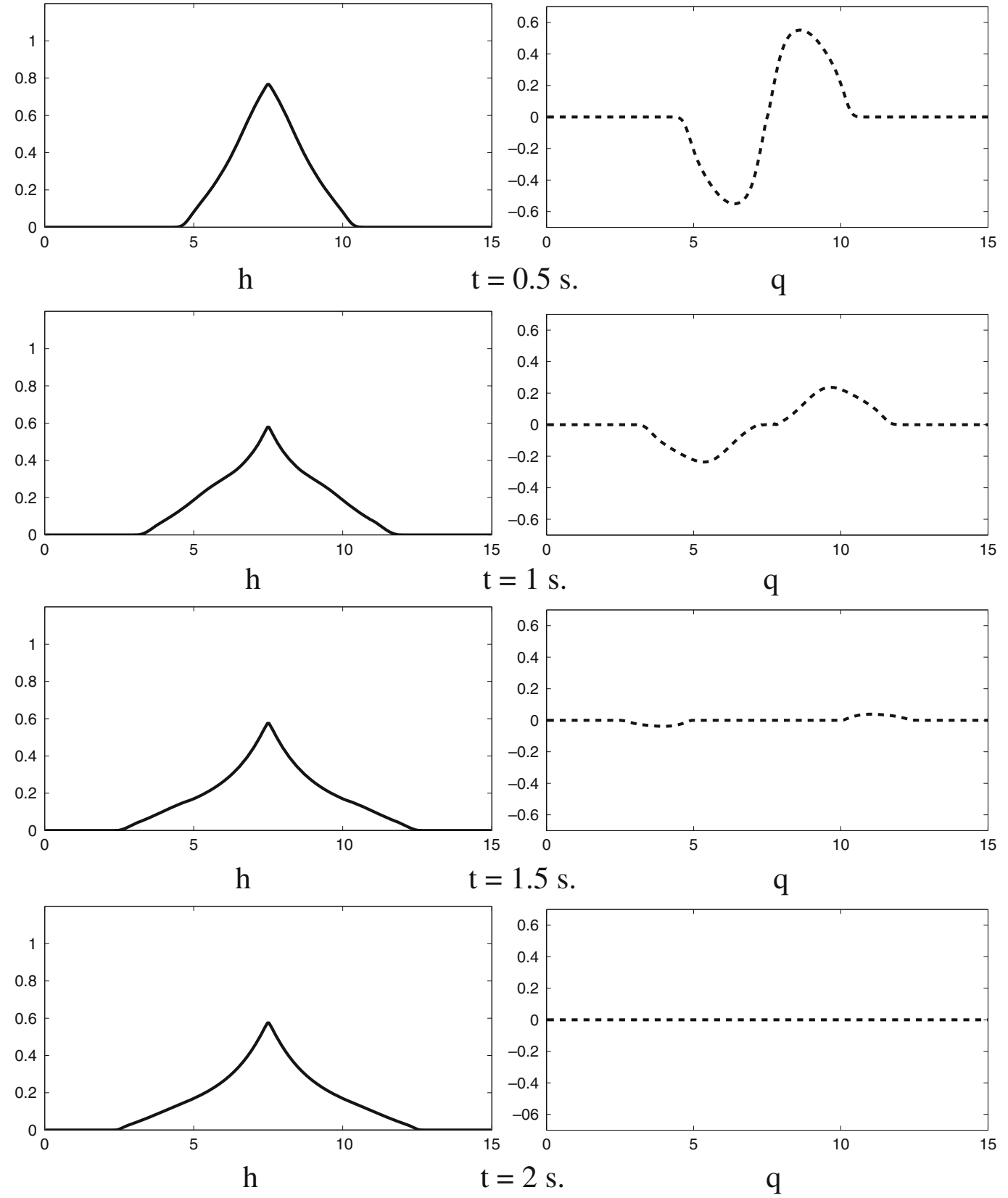

Fig. 4 Savage-Hutter model. Left: continuous line: granular free surface. Right: dashed line: discharge 


\section{Fluid-Solid Mixture Aerial Avalanches}

In most practical applications to real debris flows, the fluid which is present in the granular material can not be neglected. Recent attempts have been developed to describe mixtures of grains and fluid in shallow water two-phase or mixture models $[29,42,44]$.

The model introduced by Jackson in [30] allows to model geophysical mass flows containing a mixture of solid and fluid materials, by taking into account buoyancy effects. It is defined by the mass and momentum equations for each phase.

Let us use the following notation: subscript " $s$ " refers to the solid phase and subscript " $f$ " to the fluid one. The solid volume fraction is denoted by $\varphi$. The grain density, $\rho_{s}$ and the fluid density, $\rho_{f}$, are supposed to be constant.

Then, the two-phase model is defined by the following mass and momentum equations:

$$
\begin{aligned}
\partial_{t}\left(\rho_{s} \varphi\right)+\operatorname{div}\left(\rho_{s} \varphi \mathbf{V}_{s}\right) & =0 \\
\partial_{t}\left(\rho_{f}(1-\varphi)\right)+\operatorname{div}\left(\rho_{f}(1-\varphi) \mathbf{V}_{f}\right) & =0 \\
\rho_{s} \varphi\left(\partial_{t} \mathbf{V}_{s}+\mathbf{V}_{s} \nabla \mathbf{V}_{s}\right) & =-\operatorname{div} P_{s}+f_{0}+\rho_{s} \varphi \nabla(\mathbf{g} \cdot \mathbf{X}) \\
\rho_{f}(1-\varphi)\left(\partial_{t} \mathbf{V}_{f}+\mathbf{V}_{f} \nabla \mathbf{V}_{f}\right) & =-\operatorname{div} P_{f}-f_{0}+\rho_{f}(1-\varphi) \nabla(\mathbf{g} \cdot \mathbf{X})
\end{aligned}
$$

Where $P_{s}$ and $P_{f}$ represent the stress tensors for the solid and the fluid phase, respectively. $f_{0}$ represents the averaged value of the resultant force exerted by the fluid on a solid particle.

To obtain Jackson's model, the force $f_{0}$ is decomposed into the buoyancy force $f_{B}$ and all the remaining contributions $f$ according to [4]:

$$
f_{0}=f_{B}+f=-\varphi \nabla p_{f}+f
$$

where $p_{f}$ denotes the fluid pressure. The term $f$ collects the drag force, the lift force and the virtual mass force (see $[4,30]$ for details). Here, we assume that $f$ reduces to the drag force.

If we assume that the viscous forces related to the fluid are negligible, then the fluid stress tensor reduces to the pressure term:

$$
\nabla \cdot P_{f}=\nabla p_{f} .
$$


By taking this expression into (33c) and (33d), we obtain the system (33a), (33b) and

$$
\begin{gathered}
\rho_{s} \varphi\left(\partial_{t} \mathbf{V}_{s}+\mathbf{V}_{s} \nabla \mathbf{V}_{s}\right)=-\operatorname{div} P_{s}-\varphi \nabla p_{f}+f+\rho_{s} \nabla(\mathbf{g} \cdot \mathbf{X}), \\
\rho_{f}(1-\varphi)\left(\partial_{t} \mathbf{V}_{f}+\mathbf{V}_{f} \nabla \mathbf{V}_{f}\right)=-(1-\varphi) \nabla p_{f}-f+\rho_{f}(1-\varphi) \nabla(\mathbf{g} \cdot \mathbf{X}) .
\end{gathered}
$$

The model proposed by Pitman and Le in [44] and reformulated in [42], can be deduced following a dimensional analysis and an integration process of Jackson's model. They suppose a constant vertical profile of the velocity for the solid and the fluid phase: $U_{s}$ and $U_{f}$, respectively. Pitman-Le model can be written as follows:

$$
\begin{aligned}
\partial_{t}(h \varphi)+\partial_{X}\left(h \varphi U_{s}\right) & =0 \\
\partial_{t}(h(1-\varphi))+\partial_{X}\left(h(1-\varphi) U_{f}\right) & =0 \\
\partial_{t}\left(\varphi h U_{s}\right)+\partial_{X}\left(\varphi h U_{s}^{2}\right) & =-\frac{1}{2}(1-r) g h^{2} \cos \theta \partial_{X} \varphi \\
& -g h \cos \theta \varphi \partial_{X} h \\
& -g h \varphi \partial_{X} b \\
& +\beta h\left(U_{f}-U_{s}\right)+\mathscr{T} ; \\
\partial_{t}\left((1-\varphi) h U_{f}\right)+\partial_{X}\left((1-\varphi) h U_{f}^{2}\right) & =-g h \cos \theta(1-\varphi) \partial_{X} h \\
& -g h(1-\varphi) \partial_{X} b \\
& -\frac{1}{r} \beta h\left(U_{f}-U_{s}\right) ;
\end{aligned}
$$

where $r=\rho_{f} / \rho_{s}, \beta$ is a friction coefficient between the phases (see [44]) and $\mathscr{T}$ is the Coulomb friction term.

Let us illustrate the influence of $\varphi$ in the evolution of the avalanche. We consider first a test case consisting of a granular layer over a flat bottom whose initial profile is rectangular. The evolution of the layer is simulated by numerically solving System (37). We consider $r=0.34, \delta_{0}=25^{\circ}$ and $\varphi=0.8$. In Fig. 5 the evolution of the layer is shown. The left column shows the total height, $h_{s}+h_{f}$ (continuous line). In order to make visible the evolution of the total solid volume fraction, $h_{s}$ is also plotted in the figures (dotted line). Notice that at the front of the avalanche, the dotted line practically coincides with the continuous one, meaning that there is only 

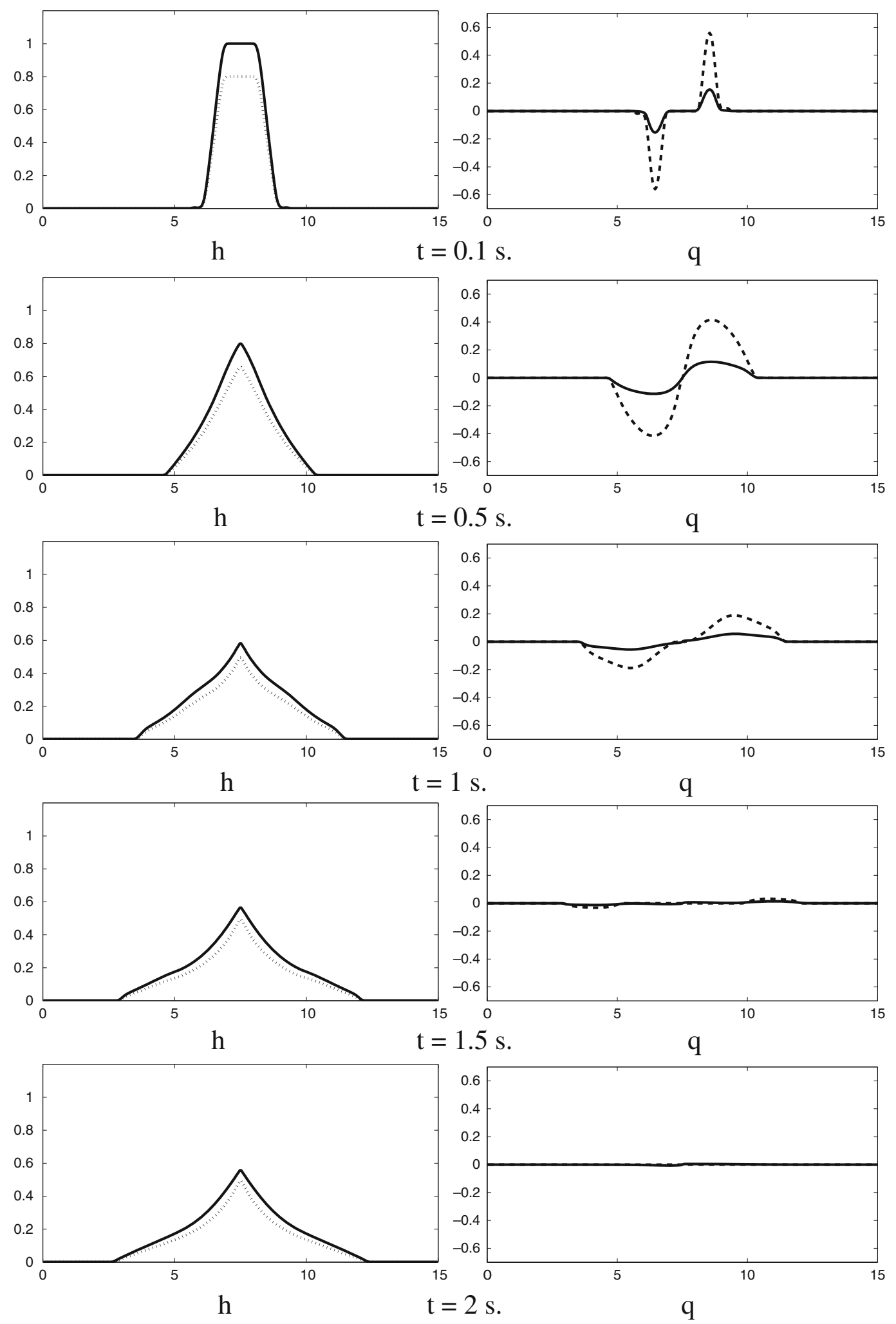

Fig. 5 Two-phase avalanche. $\varphi=0.8$. Left: continuous line: granular free surface. Dotted line: $h_{s}$; Right: discharge. Continuous line: $q_{s}$. Dashed line: $q_{f}$ 
granular material near the front. The right column shows $q_{f}$ and $q_{s}$. We can observe that the motion of the solid phase stops before. Figure 6 shows the evolution of the avalanche for $\varphi=0.4$. The initial condition and the values of $r$ and $\delta$ are the same. Let us remark that at the front the dotted line practically coincides with the horizontal axis, meaning that there is only fluid near of the front. If we compare these two simulations, we can also observe that the maximum heights and the total lengths of spreading of the avalanches are completely different. For $\varphi=0.4$, the fluid goes out the domain.

The presence of an interstitial fluid in the avalanche, neglected in the PitmanLe model, may have a strong influence in its evolution. The flow of fluidized avalanches can be much more complex than the ones simulated with the PitmanLe model. For example, non-hydrostatic pressure effects, related to the pore fluid pressure, may appear. Iverson and Denlinger extended the Savage-Hutter model in [29] to study avalanches of fluidized granular masses where the pores between the grains are assumed to be filled with a fluid, under the assumption that the velocities of both phases coincide, and by including the bed pore fluid pressure as an unknown of the system. Let us study now the derivation of a simplified version of the model proposed by Iverson and Denlinger in [29] to study partially fluidized aerial avalanches (Fig. 7).

We consider a granular layer of density $\rho_{s}$ and porosity $\psi_{0}$. We assume that the pores in the granular layer are filled with a fluid of density $\rho_{w}$. Then, the density of the fluidized layer is defined as

$$
\rho=\left(1-\psi_{0}\right) \rho_{s}+\psi_{0} \rho_{w}
$$

As in the previous section, the model will be described in local coordinates over a plain with constant slope (see Fig. 2). Again $U$ is the velocity parallel to the bottom; $W$, the velocity perpendicular to the bottom; and $\mathscr{P}$ the rotated pressure tensor.

Let us consider again the system of equations given by Euler equations in local coordinates:

$$
\left\{\begin{array}{l}
\partial_{X}(U)+\partial_{Z}(W)=0 \\
\rho \partial_{t}(U)+\rho \partial_{X}\left(U^{2}\right)+\rho \partial_{Z}(W U)-\rho \partial_{X}(\mathbf{g} \cdot \mathbf{X})=-\partial_{X}\left(\mathscr{P}_{X X}\right)-\partial_{Z}\left(\mathscr{P}_{Z X}\right), \\
\rho \partial_{t}(W)+\rho \partial_{X}(U W)+\rho \partial_{Z}\left(W^{2}\right)-\rho \partial_{Z}(\mathbf{g} \cdot \mathbf{X})=-\partial_{X}\left(\mathscr{P}_{X Z}\right)-\partial_{Z}\left(\mathscr{P}_{Z Z}\right) .
\end{array}\right.
$$

In a binary mixture model the pressure tensor of the mixture is given by

$$
\mathscr{P}=\mathscr{P}^{s}+\mathscr{P}^{f}-\rho_{w}\left(U_{f}-U_{b}\right) \otimes\left(U_{f}-U_{b}\right)-\rho_{s}\left(U_{s}-U_{b}\right) \otimes\left(U_{s}-U_{b}\right),
$$

where $U_{w}$ is the velocity of the fluid phase, $U_{s}$ is the velocity of the solid phase and $U_{b}=\left(\rho_{w} U_{w}+\rho_{w} U_{s}\right) /\left(\rho_{s}+\rho_{w}\right)$ is the barycentric velocity. 

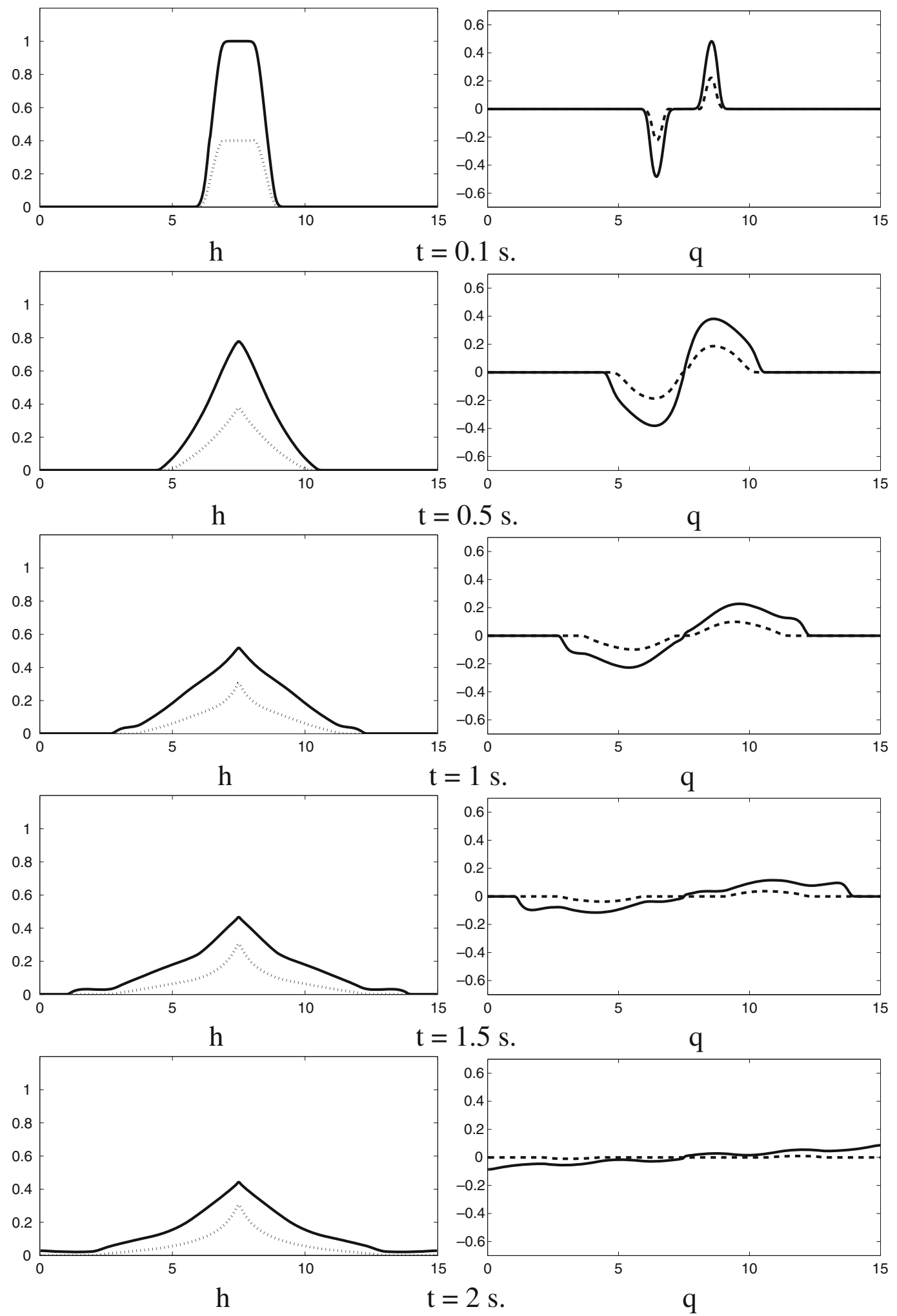

Fig. 6 Two-phase avalanche. $\varphi=0.4$. Left: continuous line: granular free surface. Dotted line: $h_{s} ;$ Right: discharge. Continuous line: $q_{s}$. Dashed line: $q_{f}$ 
Fig. 7 Partially fluidized avalanches

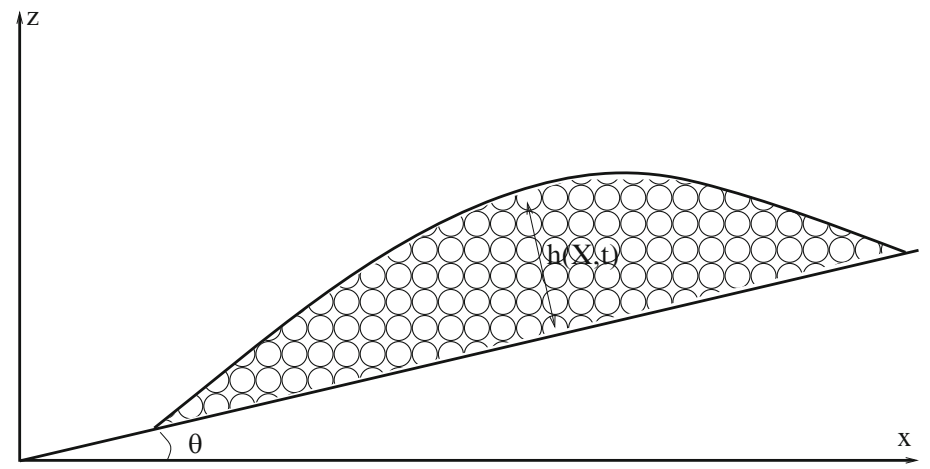

In order to model the evolution of the granular layer using the Euler equations, we suppose, following [29], that the velocity of the fluid in the pores and the grains are the same, $U_{s}=U_{f}=U$. Then $\mathscr{P}$ can be written as

$$
\mathscr{P}=\mathscr{P}^{s}+\mathscr{P}^{f}
$$

where $\mathscr{P}^{s}$ and $\mathscr{P}^{f}$ are the pressure tensor of the solid phase (grains) and the fluid phase.

The derivation of the model follows the same items as in the previous section.

\section{1 [ว] Boundary and Kinematic Conditions}

Let us denote again by $\mathbf{n}^{h}$ the unit normal vector to the free granular surface $Z=$ $h$ with positive vertical component and by $\mathbf{n}^{0}=(0,1)$ the unit normal vector to the bottom $(Z=0)$. The kinematic condition is defined by (9). For the boundary conditions, the only difference is the definition of the Coulomb friction term. The following boundary conditions are imposed:

- On $Z=h: \mathscr{P} \mathbf{n}^{h}=0$.

- On $Z=0$ : the non-penetration condition $W=0$ and the following Coulomb friction law are imposed:

$$
\mathscr{P} \mathbf{n}^{0}-\mathbf{n}^{0}\left(\mathbf{n}^{0} \cdot \mathscr{P} \mathbf{n}^{0}\right)=\left(\begin{array}{c}
-\left.\mathbf{n}^{0} \cdot\left(\mathscr{P}-\mathscr{P}^{f}\right) \mathbf{n}^{0} \frac{U}{|U|}\right|_{Z=0} \tan \delta_{0} \\
0
\end{array}\right)
$$

In this last condition, the difference between the stress tensor and the fluid stress tensor, $\mathscr{P}-\mathscr{P}^{f}$, is used to take into account the buoyancy effects, since $\mathscr{P}^{s}=$ $\mathscr{P}-\mathscr{P}^{f}$. 


\section{2 [ $[\tilde{A}]$ Dimensional Analysis}

The same non-dimensional variables as in the previous section, defined in (14), are considered. Then,

- The system of equations is defined by (15)-(17).

- The kinematic condition by (18).

- The boundary condition on $Z=h$ by (11) and (20).

- The boundary condition on $Z=0$ is different from (22). In this case we have

$$
\mu \mathscr{P}_{X Z}=-\left.\left(\mathscr{P}_{Z Z}-\mathscr{P}_{Z Z}^{f}\right) \frac{U}{|U|}\right|_{Z=0} \tan \delta_{0},
$$

and the non-penetration condition $W=0$.

\section{$3.3[1]$ Hydrostatic Pressure and Constitutive Law}

The main difference between the Savage-Hutter model presented in the previous section and the model for partially fluidized avalanches appears in this item.

From (17) we obtain

$$
\partial_{Z}\left(\mathscr{P}_{Z Z}\right)=-\rho \cos \theta+\mathscr{O}(\varepsilon)
$$

If we integrate (41) from $Z>0$ to $Z=h$, we have, up to order $\varepsilon$,

$$
\mathscr{P}_{\mathrm{ZZ}}=\rho(h-Z) \cos \theta \text {. }
$$

But, as $\mathscr{P}=\mathscr{P}^{s}+\mathscr{P}^{f}$, we have

$$
\mathscr{P}_{\mathrm{ZZ}}^{s}+\mathscr{P}_{\mathrm{ZZ}}^{f}=\mathscr{P}_{\mathrm{ZZ}}=\rho \cos \theta(h-Z) .
$$

In order to consider the anisotropy of the solid phase the following constitutive conditions are again considered (see for example [29, 45]):

$$
\mathscr{P}_{X X}^{s}=K \mathscr{P}_{\mathrm{ZZ}}^{s}, \quad \mathscr{P}_{X X}^{f}=\mathscr{P}_{\mathrm{ZZ}}^{f}
$$

where $K$ measures the anisotropy or normal stress effects in the solid phase (see previous section).

The difference appears in this step because, in order to impose these two constitutive conditions, an expression for both $\mathscr{P}_{Z Z}^{s}$ and $\mathscr{P}_{Z Z}^{f}$ has to be known. But only the expression of the total pressure $\mathscr{P}_{\mathrm{ZZ}}$ is known. 
In order to model the flow of a grain-fluid mixture, Iverson and Denlinger (see [29]) assume a linear profile of the normal stress $\mathscr{P}_{Z Z}^{f}$, which is consistent with Eq. (43). Moreover they suppose that $\mathscr{P}_{Z Z}^{f}$ takes its maximum value at $Z=0$ and is proportional to the pressure in absence of the granular phase. They suppose

$$
\mathscr{P}_{Z Z}^{f}(Z)=\lambda \rho \cos \theta(h-Z),
$$

being $\lambda$ a parameter of the model. In this case, by (43) we have

$$
\mathscr{P}_{Z Z}^{s}(Z)=(1-\lambda) \rho \cos \theta(h-Z) \text {. }
$$

Remark 1. In [29], the authors propose not to set $\lambda$ as a fixed parameter in time. Instead, they propose to rewrite the model in terms of the pore fluid pressure $p_{b e d}=\lambda \rho h \cos \theta$. Then, they assume that the evolution of $p_{b e d}$ can be described by a convection-diffusion equation. For the sake of simplicity in these notes, $\lambda$ is considered as a fixed parameter. Let us remark that we can set $\lambda=\psi_{0}$, the porosity of the layer.

\section{4 [M] Momentum Conservation Law: With Hydrostatic Pressure and Anisotropy of the Normal Stress of the Solid Phase}

By replacing (44) and (45) in (16) and using the incompressibility equation (15), we obtain up to second order

$$
\begin{aligned}
& \partial_{t}(U)+\partial_{X} U^{2}+\partial_{Z}(U W)+\partial_{X}(b+Z \cos \theta+(h-Z) \cos \theta(\lambda+K(1-\lambda))) \varepsilon \\
& \quad=-\frac{\mu}{\rho} \partial_{Z}\left(\mathscr{P}_{X Z}\right) .
\end{aligned}
$$

\section{$3.5\left[\int\right]$ Integration Process}

As in the previous section, let us define:

$$
\bar{U}=\frac{1}{h} \int_{0}^{h} U(X, Z) d Z \text { and } \overline{U^{2}}=\frac{1}{h} \int_{0}^{h} U^{2}(X, Z) d Z .
$$

As there is no difference in the integration process for the mass equation, we focus here on the momentum equation. 
Let us integrate Eq. (46) from $Z=0$ to $Z=h$ and use the kinematic conditions (18) to obtain

$$
\begin{aligned}
& \partial_{t}(h \bar{U})+\partial_{X}\left(h \overline{U^{2}}\right)+\left(\int_{0}^{h} \partial_{X}(b+Z \cos \theta+(h-Z) \cos \theta(\lambda+K(1-\lambda))) d Z\right) \varepsilon \\
& =-\frac{\mu}{\rho}\left(\mathscr{P}_{X Z}(h)-\mathscr{P}_{X Z}(0)\right) .
\end{aligned}
$$

Moreover,

$$
\begin{aligned}
& \int_{0}^{h} \partial_{X}(b+Z \cos \theta+(h-Z) \cos \theta(\lambda+K(1-\lambda))) d Z=h \partial_{X} b \\
& \quad+\partial_{X}\left(\frac{h^{2}}{2} \cos \theta(\lambda+K(1-\lambda))\right)
\end{aligned}
$$

Then, we obtain the following averaged momentum conservation law,

$$
\partial_{t}(h \bar{U})+\partial_{X}\left(h \overline{U^{2}}+\varepsilon \frac{h^{2}}{2} \cos \theta(\lambda+K(1-\lambda))\right)=-\varepsilon h \partial_{X} b-\frac{\mu}{\rho}\left(\mathscr{P}_{X Z}(h)-\mathscr{P}_{X Z}(0)\right) .
$$

Now, we can use the boundary conditions and the constitutive laws to derive $\mu \mathscr{P}_{X Z}(h)$ and $\mu \mathscr{P}_{X Z}(0)$ :

- Using (19) and the constitutive laws $\mathscr{P}_{X X}^{s}=K \mathscr{P}_{Z Z}^{s}, \mathscr{P}_{X X}^{f}=\mathscr{P}_{Z Z}^{f}$ we have

$$
\mu \mathscr{P}_{X Z}(h)=\varepsilon \partial_{X} h \mathscr{P}_{Z Z}^{s}(K-1)
$$

If we suppose again that $\mu$ is of order $\gamma$ for some $\gamma \in(0,1),\left(\mu=\tan \delta_{0}=\right.$ $\left.\mathscr{O}\left(\varepsilon^{\gamma}\right)\right)$ then

$$
\mu \mathscr{P}_{X Z}(h)=\mathscr{O}\left(\varepsilon^{1+\gamma}\right)
$$

- Using Eq. (40), we obtain

$$
\mu \mathscr{P}_{X Z}(0)=-\left.\left(\mathscr{P}_{Z Z}(0)-\mathscr{P}_{Z Z}^{f}(0)\right) \frac{U}{|U|}\right|_{Z=0} \tan \delta_{0}
$$

Now, using (42) and (43) we have

$$
\left(\mathscr{P}_{\mathrm{ZZ}}(0)-\mathscr{P}_{\mathrm{ZZ}}^{f}(0)\right)=\rho h \cos \theta(1-\lambda)+\mathscr{O}(\varepsilon)
$$


Then,

$$
\frac{\mu}{\rho} \mathscr{P}_{X Z}(0)=-\left.h \cos \theta(1-\lambda) \frac{U}{|U|}\right|_{Z=0} \tan \delta_{0}+\mathscr{O}\left(\varepsilon^{1+\gamma}\right) .
$$

Finally, substituting (49) and (50) in (48), the averaged momentum equation is obtained

$$
\begin{aligned}
& \partial_{t}(h \bar{U})+\partial_{X}\left(h \overline{U^{2}}+\varepsilon \frac{h^{2}}{2} \cos \theta(\lambda+K(1-\lambda))\right) \\
= & -\varepsilon h \partial_{X} b-\left.h(1-\lambda) \cos \theta \frac{U}{|U|}\right|_{Z=0} \tan \delta_{0}+\mathscr{O}\left(\varepsilon^{1+\gamma}\right) .
\end{aligned}
$$

\section{6 $[\hookrightarrow]$ Final System of Equations}

Coming back to the original variables, using (14), neglecting the terms of order $\varepsilon^{1+\gamma}$ and supposing a constant profile of the velocities, the following system is obtained

$$
\left\{\begin{array}{l}
\partial_{t} h+\partial_{X}(h \bar{U})=0 \\
\partial_{t}(h \bar{U})+\partial_{X}\left(h \bar{U}^{2}+g \cos \theta \frac{h^{2}}{2}(\lambda+K(1-\lambda))\right)=-g h \partial_{X} b+\mathscr{T}
\end{array}\right.
$$

where $\mathscr{T}$ is the Coulomb friction term. In this model this term must be understood as follows:

$$
\begin{aligned}
& \text { If }|\mathscr{T}| \geq \sigma_{c} \quad \Rightarrow \quad \mathscr{T}=-g h(1-\lambda) \cos \theta \frac{\bar{U}}{|\bar{U}|} \tan \delta_{0}, \\
& \text { If }|\mathscr{T}|<\sigma_{c} \quad \Rightarrow \quad \bar{U}=0,
\end{aligned}
$$

where $\sigma_{c}=g h(1-\lambda) \cos \theta \tan \delta_{0}$.

\section{Comparison with Pitman-Le Model}

Let us remark that if anisotropy is taken into account in the deduction of the solid phase momentum equation in the Pitman-Le model, Eqs. (37c) and (37d) will read as follows: 


$$
\begin{aligned}
\partial_{t}\left(\varphi h U_{s}\right)+\partial_{X}\left(\varphi h U_{s}^{2}+K g \cos \theta \varphi \frac{h^{2}}{2}\right)= & \frac{r}{2} g h^{2} \cos \theta \partial_{X} \varphi \\
& -g h \varphi \partial_{X} b \\
& +\beta h\left(U_{f}-U_{s}\right)+\mathscr{T} ;
\end{aligned}
$$

$$
\begin{aligned}
\partial_{t}\left((1-\varphi) h U_{f}\right)+\partial_{X}\left((1-\varphi) h U_{f}^{2}+g \cos \theta(1-\varphi) \frac{h^{2}}{2}\right)= & g \frac{h^{2}}{2} \cos \theta \partial_{X}(1-\varphi) \\
& -g h(1-\varphi) \partial_{X} b \\
& -\frac{1}{r} \beta h\left(U_{f}-U_{s}\right) .
\end{aligned}
$$

Let us now consider that $U_{s}=U_{f}=U$, that is, the assumption considered in the deduction of the Iverson-Denlinger model and let us define

$$
\rho=\varphi \rho_{s}+(1-\varphi) \rho_{f}
$$

Then, from (37a) and (37b) we obtain

$$
\partial_{t}(\rho h)+\partial_{X}(\rho h U)=0
$$

And from (52a) and (52b) we obtain that

$$
\partial_{t}(\rho h U)+\partial_{X}\left(\rho h U^{2}+g \cos \theta \frac{h^{2}}{2}\left(\rho+\rho_{s} \varphi(K-1)\right)\right)=-g h \rho \partial_{X} b+\mathscr{T} .
$$

Note that in the two-phase model the pressure of the fluid phase evaluated at $Z=$ 0 is

$$
p_{\text {bed }}^{P L}=g \rho_{f} \cos \theta(1-\varphi) h
$$

while in the model proposed by Iverson and Denlinger the pressure at the bottom of the fluid phase is assumed to be:

$$
p_{b e d}=\lambda \rho g \cos \theta h
$$

If we set

$$
\psi=\frac{\rho_{f}}{\rho}(1-\varphi)
$$


we have

$$
p_{\text {bed }}^{P L}=\psi \rho g \cos \theta h
$$

On the other hand, we have

$$
\rho+\rho_{s} \varphi(K-1)=\rho-\rho_{s}+\psi \frac{\rho}{r}+K\left(\rho_{s}-\psi \frac{\rho}{r}\right) .
$$

If we define finally $\lambda$ by

$$
\rho \lambda=\rho-\rho_{s}+\psi \frac{\rho}{r}
$$

we have the equality

$$
\rho+\rho_{s} \varphi(K-1)=\rho \lambda+K(\rho-\rho \lambda)
$$

Then, we can rewrite system (53) as

$$
\partial_{t}(\rho h U)+\partial_{X}\left(\rho h U^{2}+g \cos \theta \frac{h^{2}}{2}(\rho \lambda+K(\rho-\rho \lambda))\right)=-g h \rho \partial_{X} b+\mathscr{T} .
$$

That is, we have the same structure as the momentum equation of (51) for the Iverson-Denlinger model. This implies a relation between the hypothesis considered in [29] and the two-phase model when the velocities of the two phases coincide:

$$
p_{\text {bed }}^{p l}=p_{\text {bed }} r+(1-\varphi)\left(\rho_{s}-\rho_{f}\right) r g h \cos \theta
$$

Let us remark finally that, while in the Pitman-Le model the pressure of the phases are considered to be hydrostatic, the inclusion of the pressure at the bed in terms of the parameter $\lambda$ can be understood as a way to introduce a deviation from the hydrostatic pressure law in the Iverson-Denlinger model.

\section{Submarine Avalanches}

In this section, we present a simplified version of the two-layer Savage-Hutter type model proposed in [21], with application to submarine avalanches and tsunami waves generated by them.

Submarine avalanches or landslides have been poorly studied compared to their subaerial counterparts. This is however a key issue in geophysics. Indeed, submarine granular flows driven by gravity participate in the evolution of the sea bottom and in particular of the continental margins. They also represent a threat to submarine 
Fig. 8 Submarine avalanches

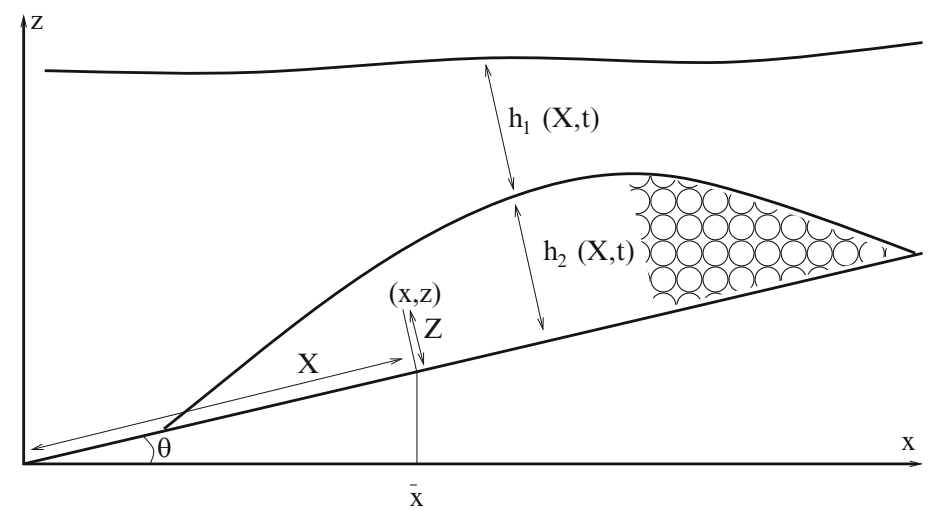

infrastructures, especially for the oil or port industries as well as to many sea shore inhabitants due to the potential tsunamis that can be triggered by such landslides.

In the model derived in this section, index 1 refers to the upper layer, composed of an homogeneous inviscid fluid of constant density $\rho_{1}$. Index 2 refers to the lower layer, composed of a granular material of density $\rho_{s}$ and porosity $\psi_{0}$ (see Fig. 8). The pores of the granular layer are assumed to be filled with the fluid of the upper layer. Accordingly, the density of layer 2 is given by:

$$
\rho_{2}=\left(1-\psi_{0}\right) \rho_{s}+\psi_{0} \rho_{1}
$$

We consider the incompressible Euler equations, with unknowns

$$
\mathbf{V}_{i}=\left(\begin{array}{c}
u_{i} \\
w_{i}
\end{array}\right), \quad i=1,2
$$

being $u_{i}$ and $w_{i}$, the horizontal and vertical velocity components of each layer, respectively. Then, the incompressible Euler equations can be written as

$$
\begin{gathered}
\operatorname{div} \mathbf{V}_{i}=0, i=1,2, \\
\rho_{i} \partial_{t} \mathbf{V}_{i}+\rho_{i} \mathbf{V}_{i} \nabla \mathbf{V}_{i}=-\operatorname{div} P_{i}+\rho_{i} \nabla(\mathbf{g} \cdot \mathbf{X}), \quad i=1,2,
\end{gathered}
$$

where $P_{i}, i=1,2$, represent the pressure tensor of each layer

$$
P_{i}=\left(\begin{array}{cc}
p_{i, x x} & p_{i, x z} \\
p_{i, z x} & p_{i, z z}
\end{array}\right), i=1,2
$$

with $p_{i, x z}=p_{i, z x}, \rho_{i}, i=1,2$, the densities of each layer, $\mathbf{X}=(x, z)$, the Cartesian coordinates and $\mathbf{g}=(0,-g)$, the gravity.

In order to model the evolution of the granular layer using the Euler equations, on the one hand we suppose following [29] (see Sect. 3) that the velocity of the fluid in the pores of the second layer coincides with that of the grains. On the other hand, $P_{2}$ is assumed to be decomposed as 


$$
P_{2}=P_{2}^{s}+P_{2}^{f}
$$

where $P_{2}^{s}$ and $P_{2}^{f}$ are the pressure tensor of the solid phase (grains) and the fluid phase, respectively.

Next, a change of variables is performed: local variables over a non-erodible bottom defined by $z=b(x)$ are considered. $X$ denotes the arc's length of the bottom and $Z$ is measured orthogonally to the bottom (see Fig. 8 and Sect. 2).

In what follows, we denote by $h_{1}$ and $h_{2}$ the thickness of the fluid and granular layers, respectively, measured orthogonally to the bottom (see Fig. 8), by $S=h_{1}+$ $h_{2}$ the free water surface. The details of this change of variables have been given in Sect. 2. Equations (55)-(56) are re-written in the new variables as follows:

$$
\left\{\begin{array}{l}
\partial_{X}\left(U_{i}\right)+\partial_{Z}\left(W_{i}\right)=0, \quad i=1,2 \\
\rho_{i} \partial_{t}\left(U_{i}\right)+\rho_{i} \partial_{X}\left(U_{i}^{2}\right)+\rho_{i} \partial_{Z}\left(W_{i} U_{i}\right)-\rho_{i} \partial_{X}(\mathbf{g} \cdot \mathbf{X}) \\
\quad=-\partial_{X}\left(\mathscr{P}_{i X X}\right)-\partial_{Z}\left(\mathscr{P}_{i X Z}\right) \quad i=1,2 \\
\rho_{i} \partial_{t}\left(W_{i}\right)+\rho_{i} \partial_{X}\left(U_{i} W_{i}\right)+\rho_{i} \partial_{Z}\left(W_{i}^{2}\right)-\rho_{i} \partial_{Z}(\mathbf{g} \cdot \mathbf{X}) \\
\quad=-\partial_{X}\left(\mathscr{P}_{i Z X}\right)-\partial_{Z}\left(\mathscr{P}_{i Z Z}\right) \quad i=1,2
\end{array}\right.
$$

where $U_{i}, i=1,2$, represent the velocity parallel to the bottom and $W_{i}, i=1,2$, the perpendicular one. The pressure tensor $\mathscr{P}_{i}$ is defined by

$$
\mathscr{P}_{i}=\left(\begin{array}{cc}
\cos \theta & \sin \theta \\
-\sin \theta & \cos \theta
\end{array}\right) P_{i}\left(\begin{array}{cc}
\cos \theta & -\sin \theta \\
\sin \theta & \cos \theta
\end{array}\right)=\left(\begin{array}{ll}
\mathscr{P}_{i, X X} & \mathscr{P}_{i, X Z} \\
\mathscr{P}_{i, Z X} & \mathscr{P}_{i, Z Z}
\end{array}\right) \text {. }
$$

Remember that, as $p_{i, x z}=p_{i, x z}$, then $\mathscr{P}_{i, X Z}=\mathscr{P}_{i, Z X}$.

Moreover, let us recall that $\rho_{1}$ is the density of the fluid and that $\rho_{2}$ is defined by (54). $\theta$ is the angle between the tangent vector of the bottom and the horizontal axis (see Fig. 8).

\section{1 [ว] Boundary and Kinematic Conditions}

Let $\mathbf{n}^{S}, \mathbf{n}^{h_{2}}$ and $\mathbf{n}^{0}=(0,1)$ be the unit normal vector to the free water surface $Z=S\left(S=h_{1}+h_{2}\right)$, to the interface $\left(Z=h_{2}\right)$ and to the bottom $(Z=0)$, respectively.

The following kinematic conditions are considered

$$
\begin{gathered}
\partial_{t} S+\left.U_{1}\right|_{Z=S} \partial_{X} S-\left.W_{1}\right|_{Z=S}=0, \\
\partial_{t} h_{2}+\left.U_{i}\right|_{Z=h_{2}} \partial_{X} h_{2}-\left.W_{i}\right|_{Z=h_{2}}=0, \quad i=1,2 .
\end{gathered}
$$


Equation (59) assumes that no water is exchanged between the two layers, which is a simplification of the model.

Finally, the following boundary conditions are imposed:

- On $Z=S$ :

$$
\mathscr{P}_{1} \mathbf{n}^{S}=0
$$

- On $Z=h_{2}$ :

$$
\begin{gathered}
\mathbf{n}^{h_{2}} \cdot\left(\mathscr{P}_{1}-\mathscr{P}_{2}\right) \mathbf{n}^{h_{2}}=0 \\
\mathscr{P}_{i} \mathbf{n}^{h_{2}}-\mathbf{n}^{h_{2}}\left(\mathbf{n}^{h_{2}} \cdot \mathscr{P}_{i} \mathbf{n}^{h_{2}}\right)=\left(\begin{array}{c}
\operatorname{fric}\left(U_{1}, U_{2}\right) \\
0
\end{array}\right) \quad i=1,2,
\end{gathered}
$$

where $\operatorname{fric}\left(U_{1}, U_{2}\right)$ is a friction term between the layers.

- On $Z=0$ :

$$
\begin{gathered}
\left(U_{2}, W_{2}\right) \cdot \mathbf{n}^{0}=0 \quad \Rightarrow \quad W_{2}=0, \\
\mathscr{P}_{2} \mathbf{n}^{0}-\mathbf{n}^{0}\left(\mathbf{n}^{0} \cdot \mathscr{P}_{2} \mathbf{n}^{0}\right)=\left(\begin{array}{c}
\left.-\left.\mathbf{n}^{0} \cdot\left(\mathscr{P}_{2}-\mathscr{P}_{1}\right) \mathbf{n}^{0} \frac{U_{2}}{\left|U_{2}\right|}\right|_{Z=0} \tan \delta_{0}\right) \\
0
\end{array}\right) .
\end{gathered}
$$

Let us remark that the term $\left(\mathscr{P}_{2}-\mathscr{P}_{1}\right)$ in the Coulomb friction law in Eq. (64) is used again in order to take into account the buoyancy effects.

\section{$5.2[\tilde{A}]$ Dimensional Analysis}

Next, a dimensional analysis of the set of Eqs. (57), the kinematic and boundary conditions is performed. The non-dimensional variables ( $\tilde{\text { }}$ ) read:

$$
\begin{aligned}
& (X, Z, t)=\left(L \tilde{X}, H \tilde{Z},(L / g)^{1 / 2} \tilde{t}\right), \\
& \left(U_{i}, W_{i}\right)=(L g)^{1 / 2}\left(\widetilde{U}_{i}, \varepsilon \widetilde{W}_{i}\right), i=1,2, \\
& h_{i}=H{\widetilde{h_{i}}}, i=1,2, \\
& \left(\mathscr{P}_{i X X}, \mathscr{P}_{i Z Z}\right)=\rho_{i} g H\left(\tilde{\mathscr{P}}_{i X X}, \tilde{\mathscr{P}}_{i Z Z}\right), i=1,2, \\
& \mathscr{P}_{i X Z}=\rho_{i} g H \mu_{i} \tilde{\mathscr{P}}_{i X Z}, \quad i=1,2,
\end{aligned}
$$


where $\mu_{1}=1, \mu_{2}=\tan \delta_{0}, \delta_{0}$ being the angle of repose in the Coulomb term (see [47]). By $L$ and $H$ we denote, respectively, the characteristic tangential and normal lengths. We suppose a shallow domain, so $\varepsilon=H / L$ is supposed to be small.

Using this change of variable, the system of Eqs. (57) are rewritten as (tildes are omitted):

$$
\begin{gathered}
\partial_{X}\left(U_{i}\right)+\partial_{Z}\left(W_{i}\right)=0, \quad i=1,2 \\
\partial_{t}\left(\rho_{i} U_{i}\right)+\rho_{i} U_{i} \partial_{X} U_{i}+\rho_{i} W_{i} \partial_{Z} U_{i}+\rho_{i} \partial_{X}\left(b+Z \cos \theta+\frac{\mathscr{P}_{i X X}}{\rho_{i}}\right) \varepsilon \\
=-\mu_{i} \partial_{Z}\left(\mathscr{P}_{i X Z}\right) \quad i=1,2, \\
\varepsilon\left\{\partial_{t}\left(\rho_{i} W_{i}\right)+\rho_{i} U_{i} \partial_{X}\left(W_{i}\right)+\rho_{i} W_{i} \partial_{Z}\left(W_{i}\right)+\partial_{X}\left(\mathscr{P}_{i X Z}\right)\right\}+ \\
+\rho_{i} \partial_{Z}(b+\cos \theta Z)=-\partial_{Z}\left(\mathscr{P}_{i Z Z}\right) \quad i=1,2 .
\end{gathered}
$$

The kinematic conditions (58)-(59) are rewritten as:

$$
\partial_{t} S+U_{1} \partial_{X} S-W_{1}=\left.0\right|_{Z=S}, \quad \partial_{t} h_{2}+U_{i} \partial_{X} h_{2}-W_{i}=\left.0\right|_{Z=h_{2}}, \quad i=1,2 .
$$

Finally, the boundary conditions (60)-(64) are now given by:

- On $Z=S$, we have $\mathbf{n}^{S}=\left(-\varepsilon \partial_{X} S, 1\right) / \varphi^{S}$ with $\varphi^{S}=\sqrt{1+\varepsilon^{2}\left(\partial_{X} S\right)^{2}}$, then from (60) we obtain

$$
\begin{aligned}
& -\varepsilon \partial_{X} S \mathscr{P}_{1 X X}+\mu_{1} \mathscr{P}_{1 Z X}=0 \\
& -\varepsilon \partial_{X} S \mu_{1} \mathscr{P}_{1 X Z}+\mathscr{P}_{1 Z Z}=0 .
\end{aligned}
$$

- On $Z=h_{2}$, we have $\mathbf{n}^{h_{2}}=\left(-\varepsilon \partial_{X} h_{2}, 1\right) / \varphi^{h_{2}}$ with $\varphi^{h_{2}}=\sqrt{1+\varepsilon^{2}\left(\partial_{X} h_{2}\right)^{2}}$, then from (61) and (62) we obtain

$$
\begin{gathered}
\mathscr{P}_{1 Z Z}=\mathscr{P}_{2 Z Z}+\mathscr{O}(\varepsilon), \\
-\varepsilon \mathscr{P}_{i X X} \partial_{X} h_{2}+\mu_{i} \mathscr{P}_{i X Z}=-\left(\mathbf{n}^{h_{2}} \mathscr{P}_{i} \mathbf{n}^{h_{2}}\right)\left(\varepsilon \partial_{X} h_{2}\right)+\operatorname{fric}\left(U_{1}, U_{2}\right), i=1,2 \\
-\varepsilon \mu_{i} \mathscr{P}_{i Z X} \partial_{X} h_{2}+\mathscr{P}_{i Z Z}=\left(\mathbf{n}^{h_{2}} \mathscr{P}_{i} \mathbf{n}^{h_{2}}\right) \quad i=1,2
\end{gathered}
$$

- On $Z=0$, we have $\mathbf{n}^{0}=(0,1)$, then from (63) and (64) we obtain

$$
\begin{gathered}
W_{2}=0 \\
\mu_{2} \mathscr{P}_{2 X Z}=-\left.\left(\mathscr{P}_{2 Z Z}-\mathscr{P}_{1 Z Z}\right) \frac{U_{2}}{\left|U_{2}\right|}\right|_{Z=0} \tan \delta_{0} .
\end{gathered}
$$




\section{3 [1] Hydrostatic Pressure and Constitutive Law}

From (68) we obtain

$$
\begin{aligned}
& \partial_{Z}\left(\mathscr{P}_{1 Z Z}\right)=-\rho_{1} \cos \theta+\mathscr{O}(\varepsilon), \\
& \partial_{Z}\left(\mathscr{P}_{2 Z Z}\right)=-\rho_{2} \cos \theta+\mathscr{O}(\varepsilon) .
\end{aligned}
$$

If we integrate (77) from $Z \geq h_{2}$ to $S$, we have, up to order $\varepsilon$,

$$
\mathscr{P}_{1 Z Z}=\rho_{1}(S-Z) \cos \theta,
$$

therefore, $\mathscr{P}_{1 \mathrm{ZZ}}\left(h_{2}\right)=\rho_{1} h_{1} \cos \theta$. Using this last expression, taking into account (72) and integrating (78) from $Z>0$ to $h_{2}$, we have

$$
\mathscr{P}_{2 Z Z}^{s}+\mathscr{P}_{2 Z Z}^{f}=\mathscr{P}_{2 Z Z}=\rho_{1} h_{1} \cos \theta+\rho_{2} \cos \theta\left(h_{2}-Z\right),
$$

up to first order. This last equation defines the total pressure, $\mathscr{P}_{2 Z Z}$, perpendicular to the bottom. The constitutive relation for both the grains and the fluid, i. e. $\mathscr{P}_{2 Z Z}^{s}$ and $\mathscr{P}_{2 Z Z}^{f}$, are required to close the model. The following relations are considered:

$$
\mathscr{P}_{1 X X}=\mathscr{P}_{1 Z Z}, \quad \mathscr{P}_{2 X X}^{s}=K \mathscr{P}_{2 Z Z}^{s}, \quad \mathscr{P}_{2 X X}^{f}=\mathscr{P}_{2 Z Z}^{f}
$$

where $K$ measures the anisotropy or normal stress effects in the solid phase (see Sect. 2).

The same difficulty found in Sect. 3 related to the definition of $\mathscr{P}_{2 \mathrm{ZZ}}^{s}$ and $\mathscr{P}_{2 \mathrm{ZZ}}^{f}$ appears here. The assumptions considered there can be adapted. We suppose

$$
\mathscr{P}_{2 \mathrm{ZZ}}^{f}(Z)=\lambda_{1} \rho_{1} h_{1} \cos \theta+\lambda_{2} \rho_{1} \cos \theta\left(h_{2}-Z\right)
$$

where $\lambda_{1}$ and $\lambda_{2}$ are two parameters. Moreover, by (80), we have

$$
\mathscr{P}_{2 Z Z}^{s}(Z)=\rho_{1} h_{1} \cos \theta\left(1-\lambda_{1}\right)+\cos \theta\left(h_{2}-Z\right)\left(\rho_{2}-\lambda_{2} \rho_{1}\right) .
$$

Remark 2. Note that if (82) and (83) are evaluated in $Z=h_{2}$, we obtain

$$
\mathscr{P}_{2 \mathrm{ZZ}}^{f}\left(h_{2}\right)=\lambda_{1} \rho_{1} h_{1} \cos \theta, \quad \mathscr{P}_{2 Z Z}^{s}\left(h_{2}\right)=\rho_{1} h_{1} \cos \theta\left(1-\lambda_{1}\right)
$$

Then, $\lambda_{1}$ controls the distribution of the pressure at the interface between the two phases of the second layer:

- A possible choice is to set $\lambda_{1}=\lambda_{2}=\psi_{0}$, where $\psi_{0}$ is the porosity of the second layer.

- We can rewrite the model in terms of $p_{\text {bed }}=\lambda_{1} \rho_{1} h_{1} \cos \theta+\lambda_{2} \rho_{1} h_{2} \cos \theta$, the pore-fluid basal pressure. In this case, a more sophisticated model can be defined 
by coupling it with a convection-diffusion equation (as proposed in [29]), as it has been mentioned in the case of partially fluidized avalanches.

By taking into account the constitutive closure equations (81) we deduce the following expression of $\mathscr{P}_{2 X X}=\mathscr{P}_{2 X X}^{s}+\mathscr{P}_{2 X X}^{f}$,

$$
\begin{gathered}
\mathscr{P}_{2 X X}=K \mathscr{P}_{2 Z Z}^{s}+\mathscr{P}_{2 Z Z}^{f} \\
=h_{1} \cos \theta \rho_{1}\left(\lambda_{1}+K\left(1-\lambda_{1}\right)\right)+\left(h_{2}-Z\right) \cos \theta\left(\lambda_{2} \rho_{1}+K\left(\rho_{2}-\lambda_{2} \rho_{1}\right)\right) .
\end{gathered}
$$

\section{$5.4[M]$ Momentum Conservation Laws: With Hydrostatic Pressure and Anisotropy of the Normal Stress of the Solid Phase of the Submerged Sediment Layer}

By replacing (79) and (84) in (5.2) and using the incompressibility equation (66), we obtain up to second order

$$
\partial_{t}\left(\rho_{1} U_{1}\right)+\rho_{1} \partial_{X} U_{1}^{2}+\rho_{1} \partial_{Z}\left(U_{1} W_{1}\right)+\rho_{1} \partial_{X}(b+S \cos \theta) \varepsilon=-\mu_{1} \partial_{Z}\left(\mathscr{P}_{1 X Z}\right),
$$

and

$$
\begin{aligned}
\partial_{t}\left(\rho_{2} U_{2}\right)+ & \rho_{2} \partial_{X} U_{2}^{2}+\rho_{2} \partial_{Z}\left(U_{2} W_{2}\right)+\rho_{2} \partial_{X}\left(b+Z \cos \theta+\frac{1}{\rho_{2}}\left[h_{1} \cos \theta \rho_{1}\left(\lambda_{1}+K\left(1-\lambda_{1}\right)\right)\right.\right. \\
& \left.\left.+\left(h_{2}-Z\right) \cos \theta\left(\lambda_{2} \rho_{1}+K\left(\rho_{2}-\lambda_{2} \rho_{1}\right)\right)\right]\right) \varepsilon=-\mu_{2} \partial_{Z}\left(\mathscr{P}_{2 X Z}\right)
\end{aligned}
$$

\section{$5.5\left[\int\right]$ Integration Process}

In this section, Eqs.(66), (85) and (86) are depth-averaged along the normal direction to the topography. Let us introduce the following notation: we denote by $\bar{U}_{i}, i=1,2$ the velocities of each layer averaged along the normal direction to the basal surface:

$$
\bar{U}_{1}=\frac{1}{h_{1}} \int_{h_{2}}^{S} U_{1}(X, Z) d Z, \quad \bar{U}_{2}=\frac{1}{h_{2}} \int_{0}^{h_{2}} U_{2}(X, Z) d Z .
$$

We also define:

$$
\overline{U_{1}^{2}}=\frac{1}{h_{1}} \int_{h_{2}}^{S} U_{1}^{2}(X, Z) d Z, \quad \overline{U_{2}^{2}}=\frac{1}{h_{2}} \int_{0}^{h_{2}} U_{2}^{2}(X, Z) d Z .
$$


If Eq. (66) is integrated from $Z=h_{2}$ to $Z=S$, we obtain

$$
0=\partial_{X}\left(h_{1} \bar{U}_{1}\right)-U_{1}(S) \partial_{X} S+W_{1}(S)+U_{1}\left(h_{2}\right) \partial_{X} h_{2}-W_{1}\left(h_{2}\right) .
$$

And using the kinematic condition (69), the following equation is derived:

$$
\partial_{t} h_{1}+\partial_{X}\left(h_{1} \bar{U}_{1}\right)=0 \text {. }
$$

Analogously, by integrating (66) between $Z=0$ and $Z=h_{2}$ we obtain

$$
0=\partial_{X}\left(h_{2} \bar{U}_{2}\right)-U_{2}\left(h_{2}\right) \partial_{X} h_{2}+W_{2}\left(h_{2}\right)-W_{2}(0),
$$

and, using the kinematic condition (69) and the boundary condition (75), the following equation is obtained:

$$
\partial_{t} h_{2}+\partial_{X}\left(h_{2} \bar{U}_{2}\right)=0
$$

If (85) is integrated from $Z=h_{2}$ to $Z=S$, we obtain

$$
\begin{gathered}
\rho_{1} \partial_{t}\left(h_{1} \bar{U}_{1}\right)+\rho_{1} \partial_{X}\left(h_{1} \overline{U_{1}^{2}}\right)-\rho_{1} U_{1}(S)\left[\partial_{t}(S)+U_{1}(S) \partial_{X} S-W_{1}(S)\right] \\
+\rho_{1} U_{1}\left(h_{2}\right)\left[\partial_{t} h_{2}+U_{1}\left(h_{2}\right) \partial_{X} h_{2}-W_{1}\left(h_{2}\right)\right]+\rho_{1}\left(\int_{h_{2}}^{S}\left(\partial_{X}(b+S \cos \theta)\right) d Z\right) \varepsilon \\
=-\mu_{1}\left(\mathscr{P}_{1 X Z}(S)-\mathscr{P}_{1 X Z}\left(h_{2}\right)\right)
\end{gathered}
$$

The expressions of $\mathscr{P}_{1 X Z}(S)$ and $\mathscr{P}_{1 X Z}\left(h_{2}\right)$ are now derived using the boundary conditions and the constitutive laws:

- Using (70) and (79) and the relation $\mathscr{P}_{1 X X}=\mathscr{P}_{1 Z Z}$ the following expression is obtained:

$$
\mu_{1} \mathscr{P}_{1 Z X}(S)=-\varepsilon \mathscr{P}_{1 X X}(S) \partial_{X} S=-\varepsilon \mathscr{P}_{1 Z Z}(S) \partial_{X} S=0+\mathscr{O}\left(\varepsilon^{2}\right)
$$

- Using (73), we have

$$
\mu_{1} \mathscr{P}_{1 X Z}\left(h_{2}\right)+\varepsilon \partial_{X} h_{2}\left(\mathscr{P}_{1 Z Z}-\mathscr{P}_{1 X X}\right)=\operatorname{fric}\left(U_{1}, U_{2}\right)+\mathscr{O}\left(\varepsilon^{2}\right)
$$

Therefore, applying the constitutive law for the fluid layer, that is, $\mathscr{P}_{1 X X}=$ $\mathscr{P}_{1 \mathrm{ZZ}}$, the following equality is derived:

$$
\mu_{1} \mathscr{P}_{1 X Z}\left(h_{2}\right)=\operatorname{fric}\left(U_{1}, U_{2}\right)+\mathscr{O}\left(\varepsilon^{2}\right)
$$

Using the kinematic condition (69), Eq. (87) and the expressions obtained for $\mu_{1} \mathscr{P}_{1 X Z}(S)(88)$ and for $\mu_{1} \mathscr{P}_{1 X Z}\left(h_{2}\right)(89)$, we obtain 
$\rho_{1} \partial_{t}\left(h_{1} \bar{U}_{1}\right)+\rho_{1} \partial_{X}\left(h_{1} \overline{U_{1}^{2}}\right)+\rho_{1}\left(\int_{h_{2}}^{S} \partial_{X}(b+S \cos \theta) d Z\right) \varepsilon=\operatorname{fric}\left(U_{1}, U_{2}\right)+\mathscr{O}\left(\varepsilon^{2}\right)$

Next, the integral term in the last equality can be computed as follows:

$$
\int_{h_{2}}^{S} \partial_{X}(b+S \cos \theta) d Z=h_{1} \partial_{X} b+\partial_{X}\left(\frac{h_{1}^{2}}{2} \cos \theta\right)+h_{1} \partial_{X}\left(\cos \theta h_{2}\right) .
$$

Finally, we obtain the equation

$$
\begin{aligned}
\rho_{1} \partial_{t}\left(h_{1} \bar{U}_{1}\right)+\rho_{1} \partial_{X}\left(h_{1} \overline{U_{1}^{2}}+\varepsilon \frac{h_{1}^{2}}{2} \cos \theta\right)= & \varepsilon \rho_{1}\left(-h_{1} \partial_{X} b-h_{1} \partial_{X}\left(\cos \theta h_{2}\right)\right) \\
& +f r i c\left(U_{1}, U_{2}\right)+\mathscr{O}\left(\varepsilon^{2}\right) .
\end{aligned}
$$

Let us now integrate Eq. (86) from $Z=0$ to $Z=h_{2}$. As in the previous case, we use the kinematic conditions (69) to obtain

$$
\begin{aligned}
& \rho_{2} \partial_{t}\left(h_{2} \bar{U}_{2}\right)+\rho_{2} \partial_{X}\left(h_{2} \overline{U_{2}^{2}}\right)+\rho_{2}\left(\int _ { 0 } ^ { h _ { 2 } } \partial _ { X } \left(b+Z \cos \theta+\frac{1}{\rho_{2}}\left[h_{1} \cos \theta \rho_{1}\left(\lambda_{1}+K\left(1-\lambda_{1}\right)\right)\right.\right.\right. \\
& \left.\left.\left.+\left(h_{2}-Z\right) \cos \theta\left(\lambda_{2} \rho_{1}+K\left(\rho_{2}-\lambda_{2} \rho_{1}\right)\right)\right]\right) d Z\right) \varepsilon=-\mu_{2}\left(\mathscr{P}_{2 X Z}\left(h_{2}\right)-\mathscr{P}_{2 X Z}(0)\right) .
\end{aligned}
$$

Let us introduce the densities ratio

$$
r=\frac{\rho_{1}}{\rho_{2}}
$$

where $\rho_{1}$ is the density of the fluid and $\rho_{2}$ is defined by (54). We obtain

$$
\begin{gathered}
\int_{0}^{h_{2}} \partial_{X}\left(b+Z \cos \theta+\frac{1}{\rho_{2}}\left[h_{1} \cos \theta \rho_{1}\left(\lambda_{1}+K\left(1-\lambda_{1}\right)\right)\right.\right. \\
\left.\left.+\left(h_{2}-Z\right) \cos \theta\left(\lambda_{2} \rho_{1}+K\left(\rho_{2}-\lambda_{2} \rho_{1}\right)\right)\right]\right) d Z=h_{2} \partial_{X} b \\
+r h_{2}\left(K\left(1-\lambda_{1}\right)+\lambda_{1}\right) \partial_{X}\left(h_{1} \cos \theta\right) \\
+\partial_{X}\left(\frac{h_{2}^{2}}{2} \cos \theta\left(r \lambda_{2}+K\left(1-r \lambda_{2}\right)\right)\right) .
\end{gathered}
$$


Replacing this last expression in (90) and dividing by $\rho_{2}$, the following equation is obtained:

$$
\begin{aligned}
& \partial_{t}\left(h_{2} \bar{U}_{2}\right)+\partial_{X}\left(h_{2} \overline{U_{2}^{2}}+\varepsilon \frac{h_{2}^{2}}{2} \cos \theta\left(r \lambda_{2}+K\left(1-r \lambda_{2}\right)\right)\right)=-\varepsilon h_{2} \partial_{X} b \\
& -\varepsilon r h_{2}\left(\lambda_{1}+K\left(1-\lambda_{1}\right)\right) \partial_{X}\left(h_{1} \cos \theta\right)-\frac{\mu_{2}}{\rho_{2}}\left(\mathscr{P}_{2 X Z}\left(h_{2}\right)-\mathscr{P}_{2 X Z}(0)\right) .
\end{aligned}
$$

Just like in the previous case, the boundary conditions and the constitutive laws are used to derive $\mu_{2} \mathscr{P}_{2 X Z}\left(h_{2}\right)$ and $\mu_{2} \mathscr{P}_{2 X Z}(0)$ :

- Using (73) and $\mathscr{P}_{2 X X}^{s}=K \mathscr{P}_{2 Z Z}^{s}, \mathscr{P}_{2 X X}^{f}=\mathscr{P}_{2 X X}^{f}$, we have

$$
\mu_{2} \mathscr{P}_{2 X Z}\left(h_{2}\right)=\operatorname{fric}\left(U_{1}, U_{2}\right)+\mu_{2} \varepsilon \partial_{X} h_{2} \mathscr{P}_{2 Z Z}^{s}(K-1) .
$$

We suppose again that $\mu_{2}=\tan \delta_{0}=\mathscr{O}\left(\varepsilon^{\gamma}\right)$ with $\gamma \in(0,1)$. Under this assumption, we have

$$
\mu_{2} \mathscr{P}_{2 X Z}\left(h_{2}\right)=\operatorname{fric}\left(U_{1}, U_{2}\right)+\mathscr{O}\left(\varepsilon^{1+\gamma}\right)
$$

- Using Eq. (76), we obtain

$$
\mu_{2} \mathscr{P}_{2 X Z}(0)=-\left.\left(\mathscr{P}_{2 Z Z}(0)-\mathscr{P}_{1 Z Z}(0)\right) \frac{U_{2}}{\left|U_{2}\right|}\right|_{Z=0} \tan \delta_{0}
$$

Now, using (79) and (80) we have

$$
\left(\mathscr{P}_{2 Z Z}(0)-\mathscr{P}_{1 Z Z}(0)\right)=h_{2} \cos \theta\left(\rho_{2}-\rho_{1}\right)+\mathscr{O}(\varepsilon)
$$

Therefore, assuming $\tan \delta_{0}=\mathscr{O}\left(\varepsilon^{\gamma}\right)$, we have

$$
\mu_{2} \mathscr{P}_{2 X Z}(0)=-\left.\left(\rho_{2}-\rho_{1}\right) h_{2} \cos \theta \frac{U_{2}}{\left|U_{2}\right|}\right|_{Z=0} \tan \delta_{0}+\mathscr{O}\left(\varepsilon^{1+\gamma}\right) .
$$

Finally, substituting (92) and (93) in (91), we derive the averaged momentum equation for the second layer

$$
\begin{aligned}
\partial_{t}\left(h_{2} \bar{U}_{2}\right)+\partial_{X}\left(h_{2} \overline{U_{2}^{2}}+\varepsilon \frac{h_{2}^{2}}{2} \cos \theta\left(r \lambda_{2}+K\left(1-r \lambda_{2}\right)\right)\right) \\
=-\varepsilon h_{2} \partial_{X} b-\varepsilon r h_{2}\left(\lambda_{1}+K\left(1-\lambda_{1}\right)\right) \partial_{X}\left(h_{1} \cos \theta\right) \\
\quad-\frac{1}{\rho_{2}} f r i c\left(U_{1}, U_{2}\right)-\left.\left((1-r) h_{2} \cos \theta+h_{2} \bar{U}_{2}^{2} d_{X} \theta\right) \frac{U_{2}}{\left|U_{2}\right|}\right|_{Z=0} \tan \delta_{0}+\mathscr{O}\left(\varepsilon^{1+\gamma}\right) .
\end{aligned}
$$




\section{$5.6[\hookrightarrow]$ Final System of Equations}

Reverting to the original variables [see (65)], neglecting the terms of order $\varepsilon^{1+\gamma}$ and supposing a constant profile of the velocities we obtain the following system:

$$
\left\{\begin{array}{l}
\partial_{t} h_{1}+\partial_{X}\left(h_{1} \bar{U}_{1}\right)=0 \\
\partial_{t}\left(h_{1} \bar{U}_{1}\right)+\partial_{X}\left(h_{1} \bar{U}_{1}^{2}+g \frac{h_{1}^{2}}{2} \cos \theta\right)= \\
\quad=-g h_{1} \partial_{X} b-g \cos \theta h_{1} \partial_{X} h_{2}+\frac{1}{\rho_{1}} f r i c\left(U_{1}, U_{2}\right) ; \\
\partial_{t} h_{2}+\partial_{X}\left(h_{2} \bar{U}_{2}\right)=0 ; \\
\partial_{t}\left(h_{2} \bar{U}_{2}\right)+\partial_{X}\left(h_{2} \bar{U}_{2}^{2}+g \frac{h_{2}^{2}}{2} \cos \theta\left(r \lambda_{2}+K\left(1-r \lambda_{2}\right)\right)\right)= \\
\quad=-g h_{2} \partial_{X} b-r g \cos \theta h_{2}\left(\lambda_{1}+K\left(1-\lambda_{1}\right)\right) \partial_{X} h_{1}-\frac{1}{\rho_{2}} f r i c\left(U_{1}, U_{2}\right)+\mathscr{T} ;
\end{array}\right.
$$

where by $\mathscr{T}$, we denote the Coulomb friction term. Again, this term must be understood as follows:

$$
\begin{aligned}
& \text { If }|\mathscr{T}| \geq \sigma_{c} \quad \Rightarrow \quad \mathscr{T}=-\left(g(1-r) h_{2} \cos \theta\right) \frac{\bar{U}_{2}}{\left|\bar{U}_{2}\right|} \tan \delta_{0}, \\
& \text { If }|\mathscr{T}|<\sigma_{c} \quad \Rightarrow \quad \bar{U}_{2}=0,
\end{aligned}
$$

where $\sigma_{c}=g\left((1-r) h_{2} \cos \theta \tan \delta_{0}\right.$. Recall that

$$
r=\frac{\rho_{1}}{\rho_{2}}
$$

where $\rho_{1}$ is the density of the fluid and $\rho_{2}$ is defined in (54). We can define the friction term between the layers fric $\left(U_{1}, U_{2}\right)$ under the following structure

$$
\operatorname{fric}\left(U_{1}, U_{2}\right)=-\mathscr{K}_{\text {in }} \cdot\left(\bar{U}_{1}-\bar{U}_{2}\right), \quad \text { with } \mathscr{K}_{\text {in }}=\rho_{1} K_{\text {in }}\left|\bar{U}_{1}-\bar{U}_{2}\right| \text {, }
$$

being $K_{\text {in }}$ a positive constant.

In Fig. 9, an example of application of the model is presented. As initial condition a rectangular granular layer is imposed at the middle of the domain. The water layer is initially at rest and its free surface is flat. As we can see in the different times shown in the figure, the avalanche produced by the granular layer interacts with the fluid and some waves appear. The stationary solution reached consists of water at rest with a flat free surface over a granular layer in equilibrium. This simulation has 

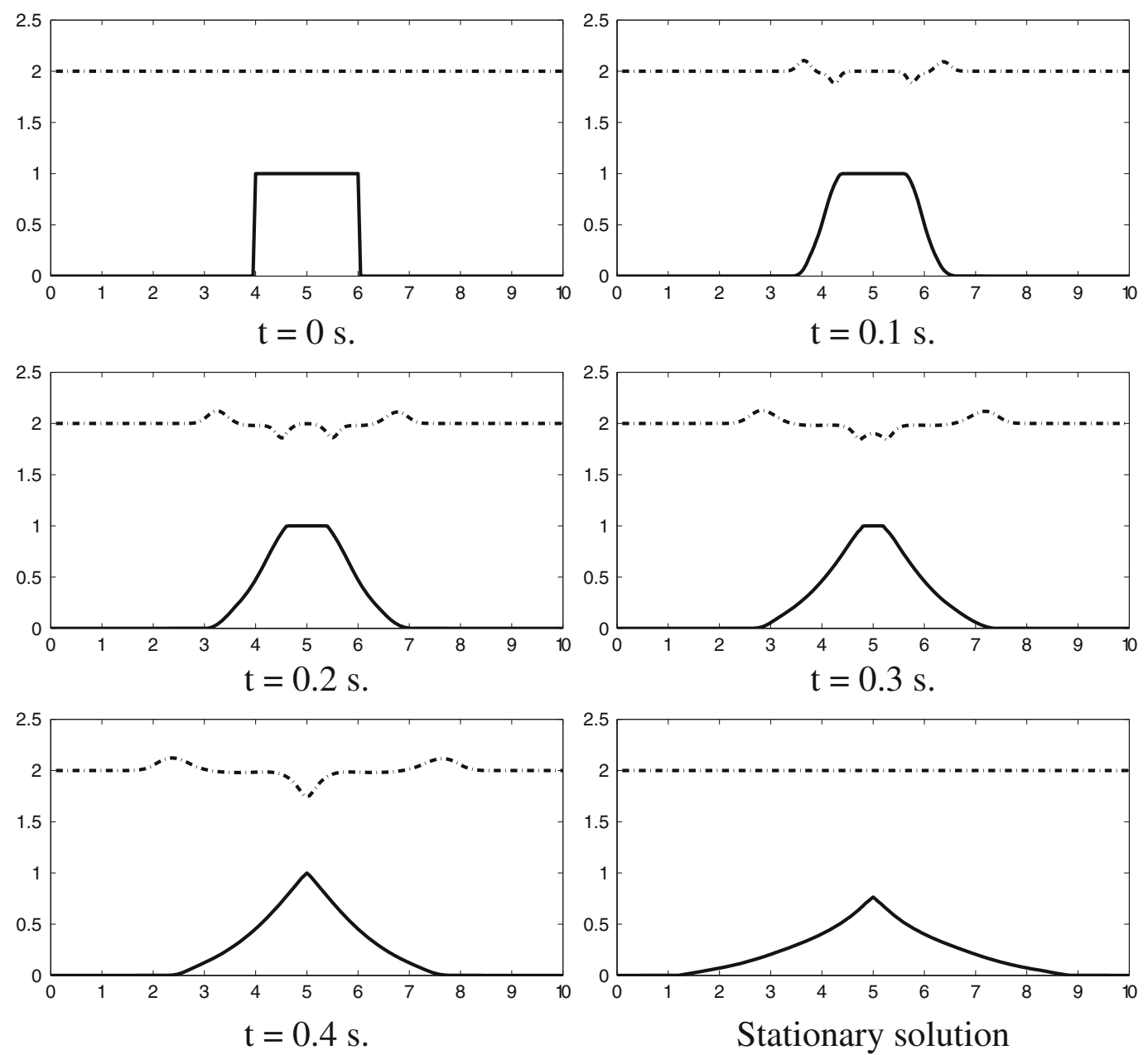

Fig. 9 Submarine avalanche test. Continuous line: granular free surface; Dashed-dotted line: water free surface

been obtained by numerically solving System (94) with the finite volume method introduced in [21]. See also [32], where an application of the model to the case of tsunamis in the Alboran Sea is studied.

\section{Entropy Inequality and Stationary Solutions of Savage-Hutter Type Models}

In this section we state without proof a result concerning the entropy inequality and the stationary solutions of the Savage-Hutter type models presented in previous sections for aerial, fluid-solid mixture and submarine avalanches. The following result can be proved for the submarine avalanche model: 
Theorem 1. System (94) has the following properties:

(i) It admits an entropy dissipation inequality,

$$
\begin{aligned}
& \partial_{t}\left(\frac{r \Lambda_{1} h_{1} \bar{U}_{1}^{2}+h_{2} \bar{U}_{2}^{2}}{2}+g b\left(r \Lambda_{1} h_{1}+h_{2}\right)+g \cos \theta \frac{r \Lambda_{1} h_{1}^{2}+\Lambda_{2} h_{2}^{2}}{2}\right. \\
& \left.\quad+g \cos \theta r \Lambda_{1} h_{1} h_{2}\right) \\
& \quad+\partial_{X}\left(r \Lambda_{1} h_{1} \bar{U}_{1}\left(\frac{\bar{U}_{1}^{2}}{2}+g b+\cos \theta\left(h_{1}+h_{2}\right)\right)+h_{2} \bar{U}_{2}\right. \\
& \left.\left(\frac{\bar{U}_{2}^{2}}{2}+g b+g \cos \theta\left(r \Lambda h_{1}+\Lambda_{2} h_{2}\right)\right)\right) \\
& \quad \leq-r K_{i n}\left|\bar{U}_{1}-\bar{U}_{2}\right|\left(U_{2}-U_{1}\right)\left(U_{2}-\Lambda_{1} U_{1}\right)-g\left((1-r) h_{2} \cos \theta\right. \\
& \left.\quad+h_{2} d_{X} \theta\left(\bar{U}_{2}^{2}-\frac{g h_{2} \cos \theta}{2}\right)\right)\left|\bar{U}_{2}\right| \tan \delta_{0} \\
& \quad+g \frac{h_{2}^{2}}{2} U_{2}\left(1-\Lambda_{2}\right) \sin \theta \partial_{X} \theta
\end{aligned}
$$

where

$$
\Lambda_{1}=\lambda_{1}+K\left(1-\lambda_{1}\right), \quad \Lambda_{2}=r \lambda_{2}+K\left(1-r \lambda_{2}\right) .
$$

(ii) It has the family of steady state solutions:

$$
\begin{gathered}
\bar{U}_{1}=0, \quad \bar{U}_{2}=0 \\
b+\left(h_{1}+h_{2}\right) \cos \theta=c s t \\
\left|\left(\Lambda_{2}-r \Lambda_{1}\right) \partial_{x}\left(b+h_{2} \cos \theta\right)+\left(1-\Lambda_{2}\right)\left(\partial_{X} b-\frac{h_{2}}{2} \sin \theta \partial_{x} \theta\right)\right| \leq(1-r) \tan \delta_{0},
\end{gathered}
$$

corresponding to water at rest over a stationary granular layer.

Note that the models presented in Sects. 2 and 3 can be seen as particular cases of this one. They can be obtained as follows:

- Savage-Hutter model: set $h_{1}=0, \bar{U}_{1}=0, \lambda_{2}=0, r=1, \lambda_{1}=1$.

- Iverson-Denlinger model: set $h_{1}=0, \bar{U}_{1}=0, \lambda_{2}=\lambda, r=1, \lambda_{1}=1$.

Therefore, the properties presented in Theorem 1 are also valid for the SavageHutter and Iverson-Denlinger models. 
Note that the stationary solutions defined by (97) correspond to situations in which the free surface of the granular layer is in equilibrium with the internal friction angle. The angle $\delta_{0}$ can be measured in laboratory experiments.

\section{Rheology of Complex Avalanches}

Several differential models have been proposed in the literature to describe sediment mixtures: a review is presented in [2]. A possible approximation is given by the model presented in Sect.3, based on a two-phase approach and a friction law proportional to the normal stress and the tangent of the internal friction angle. Another possibility is the use of visco-plastic models. They represent an approximation of the rheological behaviors of complex flows, such as debris flows, lava flows and snow avalanches.

In this section a brief introduction to non-Newtonian fluids is first given in order to motivate the definition of the stress tensor corresponding to the Herschel-Bulkley model. This model can be used to study debris flows, fluid-solid mixture avalanches.

As in the previous section we consider local coordinates on a plane slope with angle $\theta$ (see Sect. 2 for details on the notation). Let us denote the velocity vector as

$$
\mathbf{U}=\left(\begin{array}{c}
U \\
W
\end{array}\right)
$$

Let us remember that the general system of Eqs. (4) and (5) can be re-written in the new variables as follows:

$$
\left\{\begin{array}{l}
\partial_{X}(U)+\partial_{Z}(W)=0 \\
\rho \partial_{t}(U)+\rho \partial_{X}\left(U^{2}\right)+\rho \partial_{Z}(W U)-\rho \partial_{X}(\mathbf{g} \cdot \mathbf{X})=-\partial_{X}\left(\mathscr{P}_{X X}\right)-\partial_{Z}\left(\mathscr{P}_{X Z}\right) \\
\rho \partial_{t}(W)+\rho \partial_{X}(U W)+\rho \partial_{Z}\left(W^{2}\right)-\rho \partial_{Z}(\mathbf{g} \cdot \mathbf{X})=-\partial_{X}\left(\mathscr{P}_{Z X}\right)-\partial_{Z}\left(\mathscr{P}_{Z Z}\right)
\end{array}\right.
$$

where the density $\rho$ is assumed to be constant.

Let us remark that, although in these notes we are working with the negative stress tensor $\mathscr{P}$, it is also usual to write the system of equations in terms of $\sigma$, the positive Cauchy stress tensor, where

$$
\mathscr{P}=-\sigma
$$

The stress tensor is defined as the sum of the pressure component and the viscous one (cf. [9]),

$$
\sigma=-p I+\sigma^{\prime}
$$


where $I$ is the identity matrix. $\sigma^{\prime}$ is called the deviatoric part of $\sigma$. Note that we can also write,

$$
\mathscr{P}=p I-\sigma^{\prime}
$$

Let us use the notation:

$$
\sigma^{\prime}=\left(\begin{array}{cc}
\sigma_{X X}^{\prime} & \sigma_{X Z}^{\prime} \\
\sigma_{Z X}^{\prime} & \sigma_{Z Z}^{\prime}
\end{array}\right)
$$

A fluid is said to be Newtonian if $\sigma^{\prime}$ is proportional to the rate of deformation tensor $D(\mathbf{U})$, where

$$
D(\mathbf{U})=\nabla \mathbf{U}+\nabla \mathbf{U}^{t}=\left(\begin{array}{cc}
2 \partial_{X} U & \partial_{X} W+\partial_{Z} U \\
\partial_{X} W+\partial_{Z} U & 2 \partial_{Z} W
\end{array}\right)
$$

Then for a Newtonian fluid, such as water, we have

$$
\sigma^{\prime}=\eta D(\mathbf{U})
$$

where $\eta$ is the viscosity coefficient, depending on the material. In theses notes, we suppose that $\eta$ is a constant value.

Let us consider the case of a uniform flow such that

$$
D(\mathbf{U})=\left(\begin{array}{cc}
0 & \partial_{Z} U \\
\partial_{Z} U & 0
\end{array}\right) \quad \sigma^{\prime}=\left(\begin{array}{cc}
0 & \sigma^{\prime} \\
\sigma^{\prime} & 0
\end{array}\right),
$$

where $\sigma^{\prime}=\sigma_{X Z}=\sigma_{Z X}$ is the shear stress. In this case the relation which characterizes the fluid as Newtonian can be easily represented as a straight line in the plane $\left(\partial_{Z} U\right)-\left(\sigma^{\prime}\right)$ with slope $\eta$ (see Fig. 10).

The behavior of the flows of materials like honey, corn flour or paint cannot be modelled with such a linear relation. Moreover, the concept of Newtonian fluid is an idealization: there are always nonlinear relations between the shear stress and the shear rate. The study of the deviation (from the linear law) of $\sigma^{\prime}$ as a function of $D(\mathbf{U})$ belongs to the field called Rheology. The term Rheology is due to Bingham in 1929. It comes from the Greek " $\rho \varepsilon \omega "$ - "to flow". It is related to the study of deformation and flow of complex fluids.

Years around 1900s saw a significant increase of activity on these subjects, including authors like Maxwell (1868), Boltzmann (1877), Bingham, Blair, Reiner, Herschel-Bulkley, Weissenberg (all between 1900 and 1930, see [48]). Then Rheology became a research field of intense activity.

Fluids in which $\eta$ is a function of $D(\mathbf{U}), \eta=\eta(D(\mathbf{U}))$, i.e.

$$
\sigma^{\prime}=\eta(D(\mathbf{U})) D(\mathbf{U})
$$


a

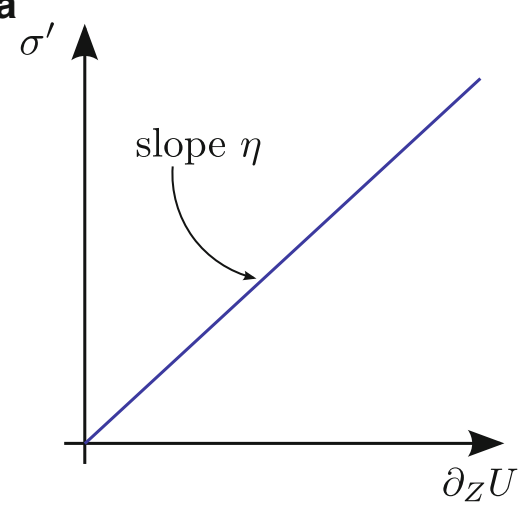

b

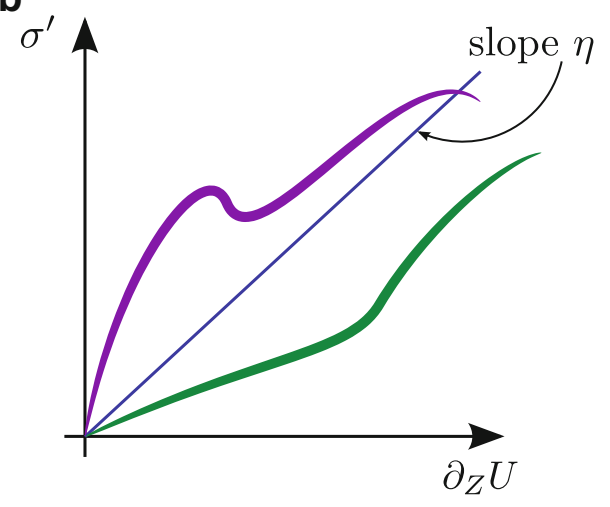

Fig. 10 (a) Representation of a Newtonian fluid in the plane $\left(\sigma^{\prime}\right)-\left(\partial_{Z} U\right)$ with viscosity $\eta$; (b) generalized Newtonian fluids

(see Fig. 10) are called Generalized Newtonian fluids; cf [41]). For example:

- Corn flour is a material whose behavior is fluid when it is gently mixed but it becomes very viscous if it is strongly mixed. That is, "viscosity" increases with "shear". Such materials are shear-thickening.

In the case of a uniform shear-thickening flow, we have $\sigma^{\prime}=\eta\left(\partial_{Z} U\right) \partial_{Z} U$, where $\eta\left(\partial_{Z} U\right)$ is an increasing function of $\partial_{Z} U$. They can be represented in the $\left(\sigma^{\prime}\right)-\left(\partial_{Z} U\right)$ plane as follows:

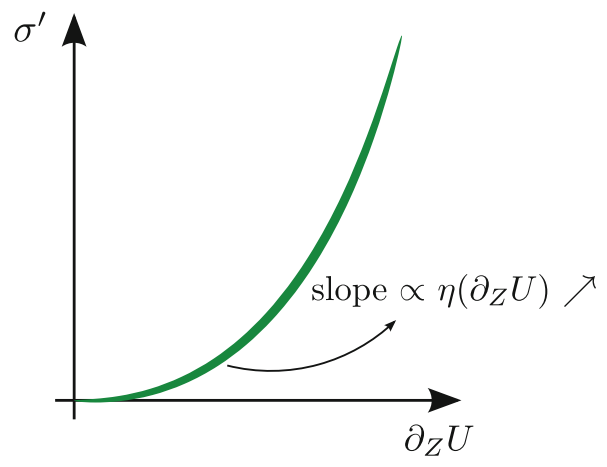

- There are many other materials, like paint, whose viscosity decreases with shear. These materials are shear-thinning.

In the case of a uniform shear-thinning flow, we have $\sigma^{\prime}=\eta\left(\partial_{Z} U\right) \partial_{Z} U$, where $\eta\left(\partial_{Z} U\right)$ is a decreasing function of $\partial_{Z} U$. They can be represented in the $\left(\sigma^{\prime}\right)-\left(\partial_{Z} U\right)$ plane as follows: 


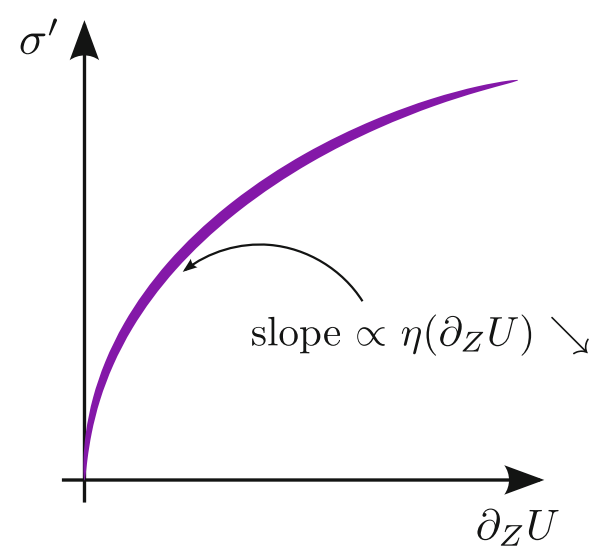

Shear-thinning and shear-thickening fluids can be modelled using power-law fluids. The viscosity of power-law fluid is defined by $\eta(D(\mathbf{U}))=\bar{\eta}|D(\mathbf{U})|^{n-1}$, for some positive constant $\bar{\eta}$ and $n \geq 0$. For these, we have

$$
\sigma^{\prime}=\bar{\eta}|D(\mathbf{U})|^{n-1} D(\mathbf{U})
$$

Let us remark that:

- If $n<1$ the material is shear-thinning.

- If $n=1$ the fluid is Newtonian and $\bar{\eta}$ is the constant viscosity.

- If $n>1$ the fluid is shear-thickening.

Nevertheless, the flow of some materials cannot be modeled by a power-law model. This is the case of clay, snow or lava that only flow when the shear stress is bigger than a critical value. These materials are example of what we can call "threshold" fluids. Below a stress $\tau_{c}$ the material present a rigid behavior but above $\tau_{c}$ the material begins to flow. They are visco-plastic materials. Bingham defined Plasticity as follows (see [10]):

We may now define plasticity as a property of solids in virtue of which they hold their shape permanently under the action of small shearing stresses but they are readily deformed, worked or molded, under somewhat larger stresses.

Bingham law follows this property and it depends on a threshold shear stress $\tau_{c}$. $\sigma^{\prime}$ is defined as follows:

$$
\begin{cases}\left|\sigma^{\prime}\right|<\tau_{c} & \text { if }|D(\mathbf{U})|=0 \\ \sigma^{\prime}=\eta D(\mathbf{U})+\tau_{c} \frac{D(\mathbf{U})}{|D(\mathbf{U})|} & \text { if }|D(\mathbf{U})| \neq 0 .\end{cases}
$$

Note that this definition implies that, in the case that $|D(\mathbf{U})|=0$, we only know that $\sigma^{\prime}$ is bounded by $\tau_{c}$. That is, $\sigma^{\prime}$ is a multivalued function in this case. It is easier to understand this law by considering the inverse function. Let us suppose that we perform two experiments of a uniform flow for a plastic material and we measure the shear rate $\partial_{Z} U$ in terms of the shear stress $\sigma^{\prime}$. We have marked with crosses in the following figure two points corresponding to the measures: 


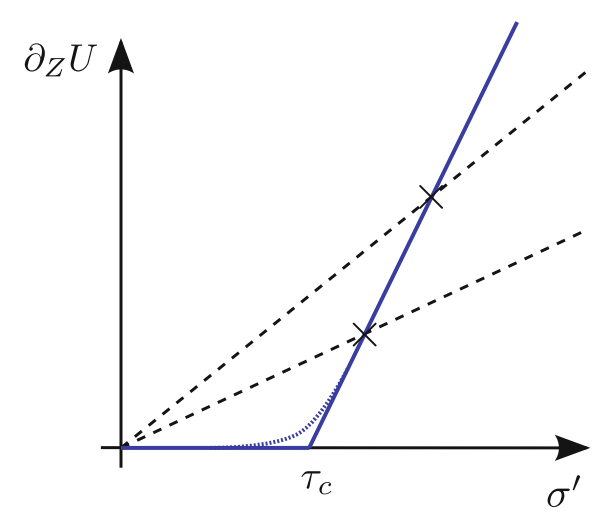

With black dashed line we plot the law corresponding to the case of Newtonian fluids, that is, straight lines passing by the point of measure and by $(0,0)$. Let us remark that if the fluid is Newtonian then it is enough to look for only one experiment in order to measure its viscosity. Remember that the viscosity in a Newtonian fluid is the inverse of the slope of such a straight line. Performing a second experiment is a way to see if the fluid is Newtonian. If we have a graph as the one of previous figure in which the second measure does not lead to a point in the same straight line, then the fluid is not Newtonian.

Conversely, if we consider a straight line passing through these two points, we obtain the value of the shear threshold $\tau_{c}$, as the point at which this line cuts the horizontal axe. Actually, measurements show that the real behavior of plastic materials does not follows exactly this straight line. They follow a curve that can be seen as a regularization of the corner around the point $\left(\tau_{c}, 0\right)$ (dashed-blue line in previous figure). The model proposed by Bingham, defined by (99), corresponds to the graph defined by the union of the two blue straight lines in the previous figure.

The general case combines power-law and plasticity. This is the HerschelBulkley constitutive equation. For the case of uniform flow it can be represented in the $\left(\sigma^{\prime}\right)-\left(\partial_{Z} U\right)$ plane as follows:

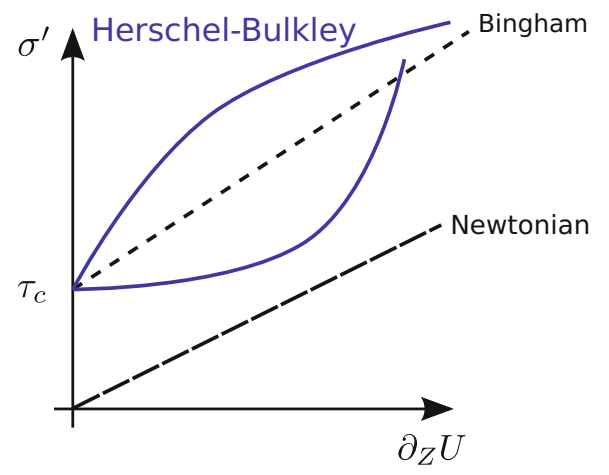

Herschel-Bulkley model is characterized by the following stress tensor:

$$
\mathscr{P}=p I-\sigma^{\prime}
$$


where

$$
\begin{cases}\sigma^{\prime}=\tau_{c} \frac{D(\mathbf{U})}{|D(\mathbf{U})|}+\bar{\eta}|D(\mathbf{U})|^{n-1} D(\mathbf{U}) & \text { if }|D(\mathbf{U})| \neq 0, \\ \left|\sigma^{\prime}\right| \leq \tau_{c} & \text { if }|D(\mathbf{U})|=0\end{cases}
$$

with $n>0$. In the case of avalanches, we have $n \in(0,1)$. In [3] the dambreak problem for visco-plastic Herschel-Bulkley fluids down a sloping flume is investigated and laboratory data are presented.

\section{A Shallow Herschel-Bulkley Model for Fluid-Solid Mixture Avalanches}

In this section, a shallow Herschel-Bulkley model is deduced (see $[1,14]$ ). One of the difficulties of Herschel-Bulkley model is that, when $|D(\mathbf{U})|=0$, only a bound of the stress tensor is known. Then, we cannot obtain a shallow HerschelBulkley model following the same steps as in the previous sections. Several types of shallow Herschel-Bulkley models have been proposed in the literature. For example in $[8,22]$ shallow visco-plastic models have been proposed in the case of nearly steady uniform regime. That is, the reference velocity for the asymptotic analysis is defined in terms of a stationary solution where the viscous contribution matches the gravity acceleration. Such a type of models are only valid for $\theta \neq 0$. In these notes, we present another type of shallow model, which corresponds to the inertial regime, where inertial and pressure-gradient terms are of the same magnitude.

As mentioned, we cannot follows exactly the same steps as in the derivation of Savage-Hutter. Basically, we cannot reproduce the items $[M]$ : the momentum conservation law and [ $\left.\int\right]$ : integration process. Nevertheless, note that the integration process in the derivation of the Savage-Hutter model is equivalent to consider the variational formulation of the model with test functions that do not depend on the vertical variable $Z$. Following this idea, we can consider first the variational formulation of Herschel-Bulkley model, which has the form of a variational inequality and then consider test functions that do not depend on the variable $Z$.

Thus, the derivation of the shallow Herschel-Bulkley model is done following the items:

- $[\partial]$ Boundary and kinematic conditions.

- $\left[\int M \cdot \psi(X, Z) \geq 0\right]$ Momentum conservation law in variational form.

- $[\tilde{A}]$ Dimensional analysis.

- $\quad\left[\int M \cdot \psi(X) \geq 0\right]$ Variational inequality for test functions independent on $Z$.

- $[\hookrightarrow]$ Final system of equations. 


\section{1 [ว] Boundary and Kinematic Conditions}

Let us remember that $\mathbf{n}^{h}$ is the unit normal vector to the free granular surface $Z=h$ with positive vertical component, and $\mathbf{n}^{0}=(0,1)$, the unit normal vector to the bottom $(Z=0)$, denoted as $\Gamma_{b}$.

The kinematic condition is considered at the free surface

$$
\partial_{t} h+\left.U\right|_{Z=h} \partial_{X} h-\left.W\right|_{Z=h}=0
$$

And the following boundary conditions are imposed:

- On $Z=h$ :

$$
\begin{aligned}
\mathbf{n}^{h} \cdot \mathscr{P} \mathbf{n}^{h} & =0 \\
\mathscr{P} \cdot \mathbf{n}^{h}-\mathbf{n}^{h}\left(\mathbf{n}^{h} \cdot \mathscr{P} \mathbf{n}^{h}\right) & =\left(\begin{array}{c}
\operatorname{fric}_{h}(U) \\
0
\end{array}\right),
\end{aligned}
$$

where $\operatorname{fric}_{h}(U)$ is the friction term between the granular layer and the air. For simplicity, we will suppose that $\operatorname{fric}_{h}(U)=0$.

- On $Z=0$ :

$$
\begin{gathered}
(U, W) \cdot \mathbf{n}^{0}=0 \quad \Rightarrow \quad W=0, \\
\mathscr{P} \mathbf{n}^{0}-\mathbf{n}^{0}\left(\mathbf{n}^{0} \cdot \mathscr{P} \mathbf{n}^{0}\right)=\left(\begin{array}{c}
-\alpha U \\
0
\end{array}\right) .
\end{gathered}
$$

That is, we consider a simple linear friction law between the material and the bottom. This is one of the main differences between the model derived in this section and the Savage-Hutter one: while in the Savage-Hutter model the Coulomb friction law controls the yielding of the material, in the shallow Herschel-Bulkley model this effect is due to the stress tensor definition and, in particular, to the rigidity coefficient $\tau_{c}$ (also called yield stress).

\section{$8.2\left[\int M \cdot \psi(X, Z) \geq 0\right]$ Momentum Conservation Law in Variational Form}

In this item, we write the variational formulation of the system defined by (98)(100). By the definition of the stress tensor $\sigma^{\prime}$ we obtain a variational inequality (see [19]).

Let us suppose that the domain filled by the avalanche, $\Omega(t)$, can be written as follows: 


$$
\Omega(t)=\left\{(X, Z) \in \mathbb{R}^{2} ; X \in[0, L] ; Z \in[0, h(X, t)]\right\} .
$$

By supposing that $h(X, t)$, the height of the avalanche, is a bounded function in space and time we may consider

$$
\mathscr{W}(t)=\left\{\Psi=(\psi, \varphi) ; \psi, \varphi \in W^{1,1+n}(\Omega(t)) /\left.\quad \psi\right|_{X=0}=\left.\psi\right|_{X=L}=0,\left.\varphi\right|_{Z=0}=0\right\}
$$

Then, we look for the solution $\mathbf{U}(t, \cdot) \in \mathscr{W}(t)$ and $p(t, \cdot) \in L^{(1+n)^{\prime}}(\Omega(t))$ satisfying for every $\Psi=(\psi, \varphi) \in \mathscr{W}(t)$ and for every $q \in L^{(1+n)^{\prime}}(\Omega(t))$ :

- The incompressibility condition:

$$
\int_{\Omega(t)} q \operatorname{div}_{(X, Z)} \mathbf{U} d X d Z=0, \quad \forall q \in L^{(1+n)^{\prime}}(\Omega(t)) .
$$

- And the momentum variational inequality:

$$
\begin{aligned}
& \int_{\Omega(t)} \rho\left(\frac{\partial \mathbf{U}}{\partial t}+\left(\mathbf{U} \cdot \nabla_{(X, Z)}\right) \mathbf{U}\right) \cdot(\Psi-\mathbf{U}) d X d Z \\
& -\int_{\Omega(t)} \rho\left(b+Z \cos \theta+\frac{p}{\rho}\right)\left(\operatorname{div}_{(X, Z)} \Psi-\operatorname{div}_{(X, Z)} \mathbf{U}\right) d X d Z \\
& +\int_{\Omega(t)} \frac{\bar{\eta}}{2}|D(\mathbf{U})|^{n-1} D(\mathbf{U}):(D(\Psi)-D(\mathbf{U})) d X d Z \\
& +\frac{\tau_{c}}{2} \int_{\Omega(t)}(|D(\Psi)|-|D(\mathbf{U})|) d X d Z \\
& +\int_{\Gamma_{b}(t)} \alpha U(\psi-U) d \gamma \geq 0 \quad \forall \Psi \in \mathscr{W}(t)
\end{aligned}
$$

with $n \in(0,1)$.

Remark 3. The inequality in the weak formulation of the momentum equation is a consequence of the weak formulation of the rigidity term in the stress tensor. If we consider the case $|D(\mathbf{U})| \neq 0$, we have

$$
\begin{aligned}
& -\int_{\Omega(t)} \operatorname{div}_{(X, Z)}\left(\sigma^{\prime}\right)(\Psi-\mathbf{U}) d X d Z=\frac{1}{2} \int_{\Omega(t)} \sigma^{\prime}: D(\Psi-\mathbf{U}) d X d Z \\
& \quad=\frac{1}{2} \int_{\Omega(t)} \tau_{c} \frac{D(\mathbf{U})}{|D(\mathbf{U})|}:(D(\Psi)-D(\mathbf{U})) d X d Z \\
& \quad+\frac{1}{2} \int_{\Omega(t)} \bar{\eta}|D(\mathbf{U})|^{n-1} D(\mathbf{U}):(D(\Psi)-D(\mathbf{U})) d X d Z .
\end{aligned}
$$


Moreover,

$$
\begin{aligned}
& \tau_{c} \int_{\Omega(t)} \frac{D(\mathbf{U})}{|D(\mathbf{U})|}:(D(\Psi)-D(\mathbf{U})) d X d Z \\
& \quad=\tau_{c} \int_{\Omega(t)} \frac{D(\mathbf{U}): D(\Psi)}{|D(\mathbf{U})|} d X d Z-\tau_{c} \int_{\Omega(t)}|D(\mathbf{U})| d X d Z
\end{aligned}
$$

and,

$$
\tau_{c} \int_{\Omega(t)} \frac{D(\mathbf{U}): D(\Psi)}{|D(\mathbf{U})|} d X d Z \leq \tau_{c} \int_{\Omega(t)}|D(\Psi)| d X d Z
$$

Observe that this is also true for the case $|D(U)|=0$. Therefore, a solution of the problem in differential form is a solution of the variational inequality. Nevertheless, it is not trivial to prove rigorously that the solution of the variational inequality is a solution of the differential problem. Although, formally it is possible to deduce the differential system from the variational inequality. One can consider as test functions $\Psi=\mathbf{U}+\lambda \mathbf{V}, \Psi=\mathbf{U}-\lambda \mathbf{V}$ and to study the limit when $\lambda$ tends to zero.

Let us develop the variational inequality (106) in terms of the components of the vector. As $\Psi=(\psi, \varphi)$ and $\mathbf{U}=(U, W)$, we have

$$
\begin{aligned}
& \int_{\Omega(t)} \rho\left(\partial_{t}(U)+\rho U \partial_{X} U+\rho W \partial_{Z} U\right)(\psi-U) d X d Z \\
+ & \int_{\Omega(t)} \rho\left(\partial_{t}(W)+\rho U \partial_{X} W+\rho W \partial_{Z} W\right)(\varphi-W) d X d Z \\
- & \int_{\Omega(t)} \rho\left(b+Z \cos \theta+\frac{p}{\rho}\right)\left(\partial_{X}(\psi-U)+\partial_{Z}(\varphi-W)\right) d X d Z \\
+ & \int_{\Omega(t)} \bar{\eta}|D(\mathbf{U})|^{n-1}\left(2 \partial_{X}(U) \partial_{X}(\psi-U)\right. \\
+ & \left.\left(\partial_{X}(W)+\partial_{Z}(U)\right)\left(\partial_{X}(\varphi-W)+\partial_{Z}(\psi-U)\right)+2 \partial_{Z}(W) \partial_{Z}(\varphi-W)\right) d X d Z \\
+ & \tau_{c} \int_{\Omega(t)}\left(\sqrt{\left(\partial_{X} \psi\right)^{2}+\frac{1}{2}\left(\partial_{X} \varphi+\partial_{Z} \psi\right)^{2}+\left(\partial_{Z} \varphi\right)^{2}}\right. \\
- & \left.\sqrt{\left(\partial_{X} U\right)^{2}+\frac{1}{2}\left(\partial_{X} W+\partial_{Z} U\right)^{2}+\left(\partial_{Z} W\right)^{2}}\right) d X d Z \\
+ & \int_{\Gamma_{b}} \alpha U(\psi-U) d \gamma \geq 0
\end{aligned}
$$


where

$$
|D(\mathbf{U})|^{n-1}=\left(4\left(\partial_{X} U\right)^{2}+2\left(\partial_{X} W+\partial_{Z} U\right)^{2}+4\left(\partial_{Z} W\right)^{2}\right)^{(n-1) / 2} .
$$

\section{$8.3[\tilde{A}]$ Dimensional Analysis}

Next, a dimensional analysis of the set of Eqs. (98), the kinematic and boundary conditions is performed. The non-dimensional variables ( $\tilde{.}$ ) read:

$$
\begin{aligned}
& (X, Z, t)=\left(L \tilde{X}, H \tilde{Z},(L / g)^{1 / 2} \tilde{t}\right), \\
& (U, W)=(L g)^{1 / 2}(\tilde{U}, \varepsilon \tilde{W}) \\
& h=H \tilde{h} \\
& \alpha=\varepsilon(L g)^{1 / 2} \tilde{\alpha} \\
& p=g H \tilde{p} \\
& \tau_{c}=g H \tilde{\tau_{c}} \\
& \bar{\eta}=H g^{\left(1-\frac{n}{2}\right)} L^{\frac{n}{2}} \tilde{\bar{\eta}} .
\end{aligned}
$$

Then,

$$
\sigma^{\prime}=g H \widetilde{\sigma^{\prime}}=g H\left(\begin{array}{ll}
{\widetilde{\sigma^{\prime}}}_{\tilde{X}} \tilde{X} & {\widetilde{\sigma^{\prime}}}_{\tilde{X} \tilde{Z}} \\
{\widetilde{\sigma^{\prime}}}_{\tilde{X} \tilde{Z}} & {\widetilde{\sigma^{\prime}}}_{\tilde{Z} \tilde{Z}}^{\prime}
\end{array}\right)
$$

With

$$
\begin{cases}\widetilde{\sigma^{\prime}}=\widetilde{\tau}_{c} \frac{\tilde{D}_{\varepsilon}(\tilde{\mathbf{U}})}{\left|\widetilde{D_{\varepsilon}}(\tilde{\mathbf{U}})\right|}+\tilde{v} \tilde{D}_{\varepsilon}(\tilde{\mathbf{U}}) & \text { if }\left|\tilde{D}_{\varepsilon}(\tilde{\mathbf{U}})\right| \neq 0 \\ \left|\widetilde{\sigma^{\prime}}\right| \leq \widetilde{\tau_{c}} & \text { if }\left|\tilde{D}_{\varepsilon}(\tilde{\mathbf{U}})\right|=0\end{cases}
$$

And

$$
\tilde{D}_{\varepsilon}(\tilde{\mathbf{U}})=\left(\begin{array}{cc}
2 \partial_{\tilde{X}} \tilde{U} & \varepsilon \partial_{\tilde{X}} \tilde{W}+\frac{1}{\varepsilon} \partial_{\tilde{Z}} \tilde{U} \\
\varepsilon \partial_{\tilde{X}} \tilde{W}+\frac{1}{\varepsilon} \partial_{\tilde{Z}} \tilde{U} & 2 \partial_{\tilde{Z}} \tilde{W}
\end{array}\right) .
$$


Using the above change of variables, the system of Eqs. (98) can be also rewritten as follows (tildes are again omitted):

$$
\begin{gathered}
\partial_{X}(U)+\partial_{Z}(W)=0 \\
\partial_{t}(\rho U)+\rho U \partial_{X} U+\rho W \partial_{Z} U+\rho \partial_{X}\left(b+Z \cos \theta+\frac{p}{\rho}\right) \varepsilon=\varepsilon \partial_{X}\left(\sigma_{X X}^{\prime}\right)+\partial_{Z}\left(\sigma_{X Z}^{\prime}\right) \\
\varepsilon\left\{\partial_{t}(\rho W)+\rho U \partial_{X}(W)+\rho W \partial_{Z}(W)-\partial_{X}\left(\sigma_{X Z}^{\prime}\right)\right\}+ \\
+\rho \partial_{Z}(b+\cos \theta Z)=-\partial_{Z}(p)+\partial_{Z}\left(\sigma_{Z Z}^{\prime}\right) .
\end{gathered}
$$

For the momentum variational inequality (107), we also consider the following nondimensional test functions:

$$
\Psi=(\psi, \varphi)=(L g)^{1 / 2}(\tilde{\psi}, \varepsilon \tilde{\varphi})
$$

The variational inequality (107) is rewritten as (tildes are omitted again):

$$
\begin{aligned}
& \int_{\Omega(t)} \rho\left(\partial_{t}(U)+\rho U \partial_{X} U+\rho W \partial_{Z} U\right)(\psi-U) d X d Z \\
+ & \varepsilon^{2} \int_{\Omega(t)} \rho\left(\partial_{t}(W)+\rho U \partial_{X} W+\rho W \partial_{Z} W\right)(\varphi-W) d X d Z \\
+ & \int_{\Omega(t)} \rho \varepsilon\left(\partial_{X} b(\psi-U)+\cos \theta(\varphi-W)\right) d X d Z \\
& -\int_{\Omega(t)} \rho \varepsilon \frac{p}{\rho}\left(\partial_{X}(\psi-U)+\partial_{Z}(\varphi-W)\right) d X d Z \\
+ & \int_{\Omega(t)} \bar{\eta}\left|D_{\varepsilon}(\mathbf{U})\right|^{n-1}\left(2 \varepsilon \partial_{X}(U) \partial_{X}(\psi-U)+\varepsilon\left(\varepsilon \partial_{X}(W)+\frac{1}{\varepsilon} \partial_{Z}(U)\right)\left(\varepsilon \partial_{X}(\varphi-W)\right.\right. \\
+ & \left.\frac{1}{\varepsilon} \partial_{Z}(\psi-U)\right) \\
+ & \left.+2 \varepsilon \partial_{Z}(W) \partial_{Z}(\varphi-W)\right) d X d Z \\
+ & \int_{\Gamma_{b}} \alpha \int_{\Omega(t)} \varepsilon\left(\sqrt{\left(\partial_{X} \psi\right)^{2}+\frac{1}{2}\left(\varepsilon \partial_{X} \varphi+\frac{1}{\varepsilon} \partial_{Z} \psi\right)^{2}+\left(\partial_{Z} \varphi\right)^{2}}\right. \\
& \sqrt{\left.\left(\partial_{X} U\right)^{2}+\frac{1}{2}\left(\varepsilon \partial_{X} W+\frac{1}{\varepsilon} \partial_{Z} U\right)^{2}+\left(\partial_{Z} W\right)^{2}\right) d X d Z} \\
& +U) d \gamma \geq 0
\end{aligned}
$$


where

$$
\left|D_{\varepsilon}(\mathbf{U})\right|^{n-1}=\left(4\left(\partial_{X} U\right)^{2}+2\left(\varepsilon \partial_{X} W+\frac{1}{\varepsilon} \partial_{Z} U\right)^{2}+4\left(\partial_{Z} W\right)^{2}\right)^{(n-1) / 2} .
$$

Let us also remark that for the inclined plane case considered in these notes we have

$$
\partial_{X} b=\sin \theta
$$

\section{$8.4\left[\int M \cdot \psi(X) \geq 0\right]$ Variational Inequality for Test Functions Independent of $Z$}

In this section, we obtain the mass and momentum equations of a Shallow HerschelBulkley model. To obtain it, we neglect the second order terms $\left(\mathscr{O}\left(\varepsilon^{2}\right)\right)$ and we consider test functions which are independent of $Z$.

Let us remark that to consider test functions independent of $Z$ is analogous to depth average the mass and momentum equations. In fact, we can see that if $\tau_{c}=0$, then we have a variational equality for the momentum conservation and the procedure described below is another way of deriving the Shallow Water equations. And, if the Coulomb friction law is considered at the bottom, the Savage-Hutter model deduced in Sect. 2 is recovered.

First, note that if $q \in L^{(1+n)^{\prime}}(\Omega(t))$ is independent of $Z$ then

$$
\int_{\Omega(t)} q \operatorname{div}_{(X, Z)} \mathbf{U}=\int_{0}^{L} q(X)\left(\int_{0}^{h} \operatorname{div}_{(X, Z)} \mathbf{U} d Z\right) d X=0 .
$$

By using the kinematic conditions, we obtain

$$
\int_{0}^{L} q(X)\left(\partial_{t} h+\partial_{X}(h U)\right) d X=0, \quad \forall q \in L^{(1+n)^{\prime}}([0, L]) .
$$

This gives a different way to obtain the mass conservation equation:

$$
\partial_{t} h+\partial_{X}(h U)=0
$$

Let us now consider test functions $\Psi=(\psi, \varphi)$ where $\psi$ is independent of $Z$. Analogously to previous sections, we assume that the velocity parallel to the bottom $U$ is independent of $Z$. Then, if we neglect second order terms $\left(\mathscr{O}\left(\varepsilon^{2}\right)\right)$ in (112), we obtain 


$$
\begin{aligned}
& \int_{0}^{L} \rho h\left(\partial_{t}(U)+\rho U \partial_{X} U\right)(\psi-U) d X d Z \\
+ & \int_{\Omega(t)} \rho \varepsilon\left(\partial_{X} b(\psi-U)+\cos \theta(\varphi-W)\right) d X d Z \\
- & \int_{\Omega(t)} \rho \varepsilon \frac{p}{\rho}\left(\partial_{X}(\psi-U)+\partial_{Z}(\varphi-W)\right) d X d Z \\
+ & \int_{\Omega(t)} \bar{\eta} 2^{2 n-2}\left(\left(\partial_{X} U\right)^{2}+\left(\partial_{Z} W\right)^{2}\right)^{n-1}\left(2 \varepsilon \partial_{X}(U) \partial_{X}(\psi-U)\right. \\
+ & \left.2 \varepsilon \partial_{Z}(W) \partial_{Z}(\varphi-W)\right) d X d Z \\
+ & \tau_{c} \int_{\Omega(t)} \varepsilon\left(\sqrt{\left(\partial_{X} \psi\right)^{2}+\left(\partial_{Z} \varphi\right)^{2}}-\sqrt{\left(\partial_{X} U\right)^{2}+\left(\partial_{Z} W\right)^{2}}\right) d X d Z \\
+ & \int_{0}^{L} \alpha U(\psi-U) d \gamma \geq 0 .
\end{aligned}
$$

Moreover, by using the incompressibility condition, by choosing also test functions with zero divergence whose vertical component vanishes at the bottomto be consistent with boundary condition (104)—we have

$$
W=-Z \partial_{X} U, \quad \text { and } \quad \varphi=-Z \partial_{X} \psi
$$

By using (114) we get:

$$
\int_{\Omega(t)}\left(\partial_{X} b(\psi-U)+\cos \theta(\varphi-W)\right) d X d Z=\int_{0}^{L}\left(h \partial_{X} b+\partial_{X}\left(\frac{h^{2}}{2} \cos \theta\right)\right)(\psi-U) d X .
$$

Finally, using this last equality and (114), we obtain from (113):

$$
\begin{aligned}
& \int_{0}^{L} \rho h\left(\partial_{t}(U)+\rho U \partial_{X} U\right)(\psi-U) d X d Z \\
+ & \int_{0}^{L} \rho\left(h \partial_{X} b+\partial_{X}\left(\frac{h^{2}}{2} \cos \theta\right)\right)(\psi-U) d X \\
+ & \int_{0}^{L} 2^{3 n-1} \bar{\eta}\left|\partial_{X} U\right|^{n-1} \varepsilon \partial_{X}(U) \partial_{X}(\psi-U) d X \\
+ & 2 \tau_{c} \int_{0}^{L} \varepsilon h\left(\left|\partial_{X} \psi\right|-\left|\partial_{X} U\right|\right) d X \\
+ & \int_{0}^{L} \alpha U(\psi-U) d \gamma \geq 0 .
\end{aligned}
$$




\section{$8.5[\hookrightarrow]$ Final System of Equations}

Coming back to the original variables and using (108), we obtain the following system:

- Mass conservation:

$$
\int_{0}^{L}\left(\partial_{t} h+\partial_{X}(h U)\right) q(X) d X=0, \quad \forall q \in L^{(1+n)^{\prime}}([0, L]) .
$$

- Momentum variational inequality: $\forall \psi \in W^{1,1+n}([0, L])$,

$$
\begin{aligned}
& \int_{0}^{L} \rho\left(h \partial_{t}(U)+\rho h U \partial_{X} U+g h \partial_{X} b+g \partial_{X}\left(\frac{h^{2}}{2} \cos \theta\right)\right)(\psi-U) d X d Z \\
+ & \int_{0}^{L} 2^{3 n-1} \bar{\eta}\left|\partial_{X} U\right|^{n-1} \partial_{X}(U) \partial_{X}(\psi-U) d X \\
+ & 2 \tau_{c} \int_{0}^{L} h\left(\left|\partial_{X} \psi\right|-\left|\partial_{X} U\right|\right) d X \\
+ & \int_{0}^{L} \alpha U(\psi-U) d \gamma \geq 0
\end{aligned}
$$

Note that the first line corresponds to convection and pressure terms in Shallow Water systems and the second one to viscous effects. The third line contains the terms associated to the rigidity properties of the material. Last line of previous equation correspond to the bottom friction term.

Let us remark that (116) and (117) corresponds to the weak formulation of the following partial differential system:

$$
\left\{\begin{array}{l}
\partial_{t} h+\partial_{X}(h U)=0 \\
h\left(\partial_{t} U+U \partial_{x} U+g(b+h \cos \theta)\right)+\alpha U-\partial_{X}\left(h \sigma^{\prime}\right)=0
\end{array}\right.
$$

where

$$
\begin{cases}\sigma^{\prime}=2^{3 n-1} \bar{\eta}\left|\partial_{X} U\right|^{n-1} \partial_{X} U+2 \tau_{c} \frac{\partial_{X} U}{\left|\partial_{X} U\right|} & \text { if }\left|\partial_{x} U\right| \neq 0 \\ \left|\sigma^{\prime}\right| \leq 2 \tau_{c} & \text { if }\left|\partial_{x} U\right|=0 .\end{cases}
$$

As mentioned before, it is easy to see that if $\tau_{c}=0, n=1$ and the linear friction law is replaced by the Coulomb friction law (13) then the Savage-Hutter model deduced in Sect. 2-with standard viscous terms-is obtained. 


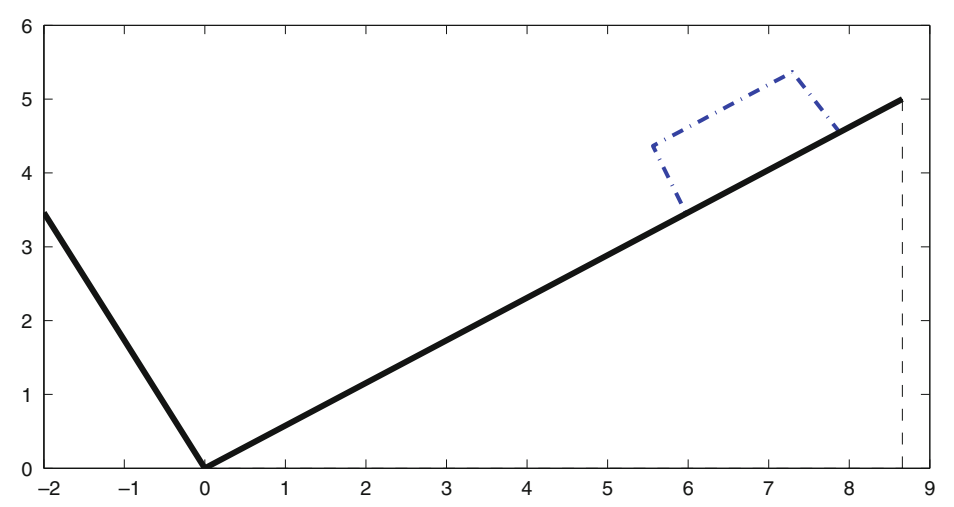

Fig. 11 Initial condition. Dashed-dotted line: free surface of the viscoplastic material. Continuous line: bottom

We refer to [1] for the discretization of this shallow-Herschel-Bulkley model. Let us present an example. As initial condition a rectangular layer is considered on a closed domain with a plain with a slope of $30^{\circ}$ (see Fig. 11).

Figure 12 shows the evolution of the avalanche of the visco-plastic material corresponding to $n=1, \tau_{c}=4, \bar{\eta}=10^{-2}, \alpha=10^{-2}$. The left column shows the evolution of the free surface at times $t \in\{0.4,1,2.4,4\} \mathrm{s}$. The right column shows the velocity profile. This simulation shows a typical behavior of visco-plastic fluids: at the beginning it moves as a rigid body and then it starts to flow as a viscous fluid. Indeed, notice that for $t=0.4$ and $t=1 \mathrm{~s}$ the velocity profile is nearly constant on all the domain filled by the avalanche, but this is no more the case for $t=2.4 \mathrm{~s}$. For $t=4 \mathrm{~s}$. the material is at rest. These different behaviors are due to the definition of the stress tensor (119).

\section{Appendix: Bed-Load Sediment Transport Formulae}

In this appendix we present several possible definitions of the solid transport discharge, $q_{b}$, that allow one to close the Saint-Venant Exner system (see Sect. 1).

The study of the definition of the solid transport discharge can be seen as a deterministic problem or a probabilistic one. For example, deterministic methods have been proposed by Meyer-Peter \& Müller [36] and probabilistic methods by Einstein [20].

In general, the models take into account the fact that motion of the granular sediment begins when the shear stress $(\tau)$ is bigger than a certain critical shear stress $\left(\tau_{c}\right)$. Moreover, shear stress can be written in terms of the hydrodynamic unknowns $h$ and $u$ by

$$
\tau=\gamma R_{h}\left|S_{f}\right|
$$



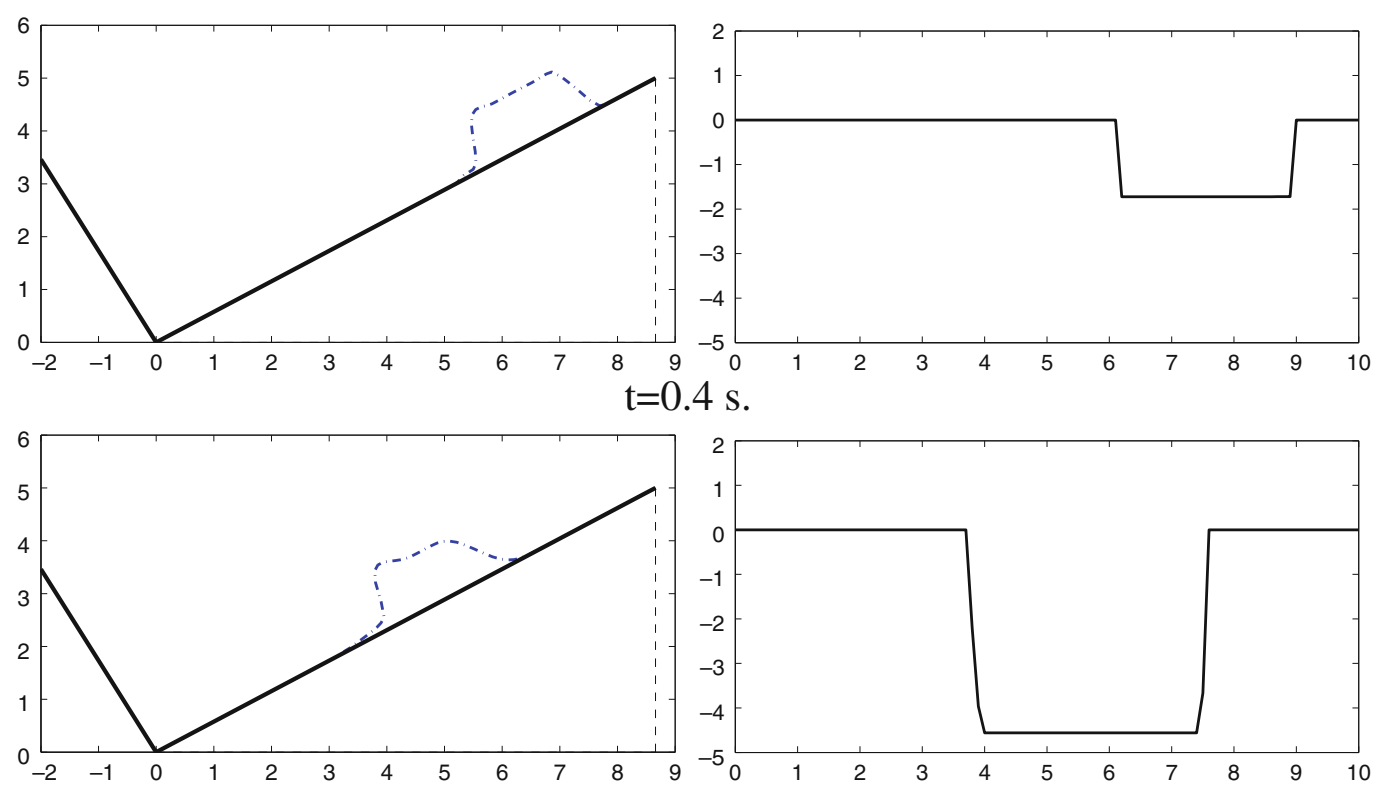
$\mathrm{t}=1 \mathrm{~s}$.

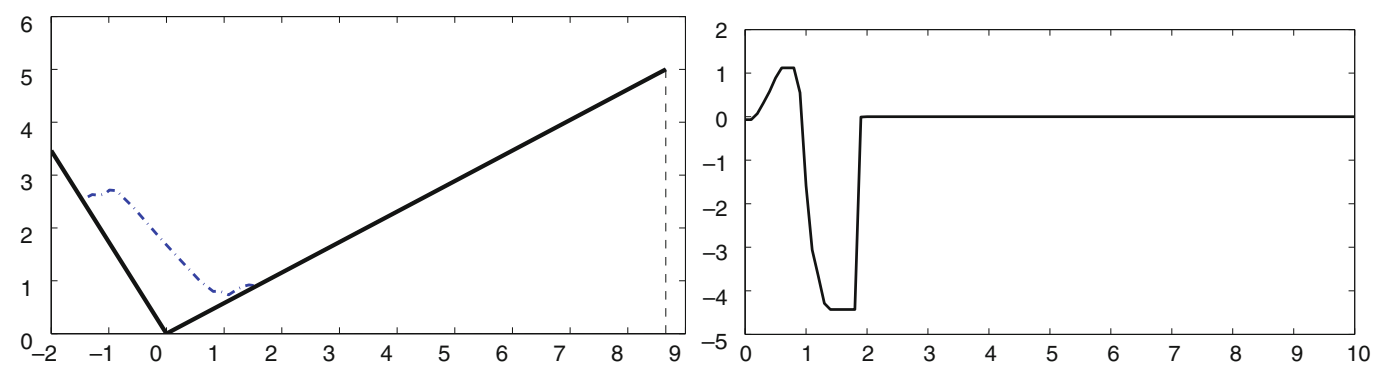

$\mathrm{t}=2.4 \mathrm{~s}$.

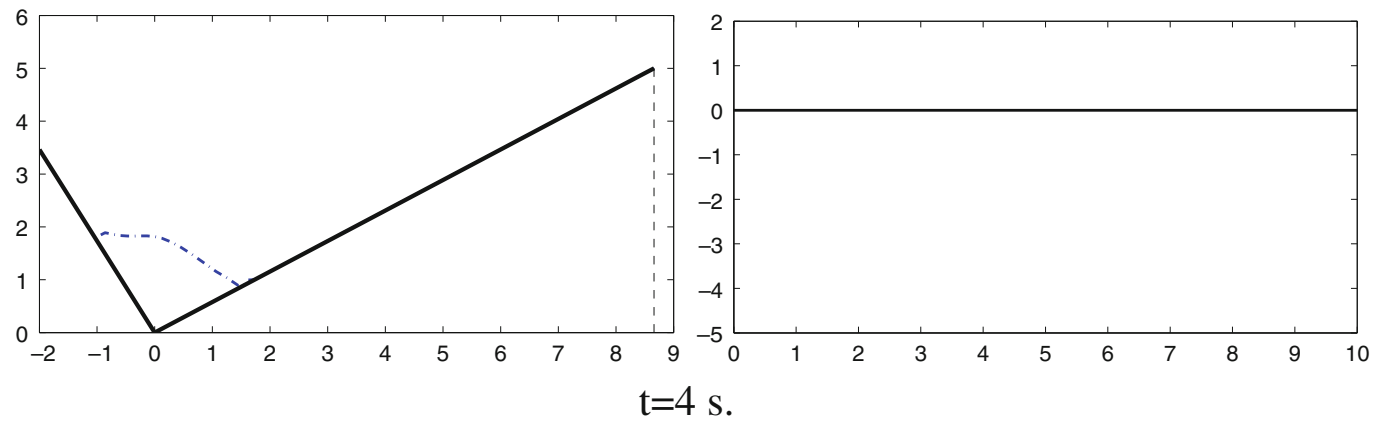

Fig. 12 Complex avalanche: Herschel-Bulkley model. Left: free surface; Right: velocity

Here $S_{f}$ is defined by (2) and $\gamma$ is the specific weight of fluid $\gamma=g \rho_{w}$, where $\rho_{w}$ is the water density.

Shear stress appears usually in non-dimensional form in the formula of $q_{b}$. If $\tau_{*}$ and $\tau_{* c}$ represent the non-dimensional shear stress and the critical shear stress, respectively, then 


$$
\tau_{*}=\frac{\tau}{\left(\gamma_{s}-\gamma\right) d}, \quad \tau_{* c}=\frac{\tau_{c}}{\left(\gamma_{s}-\gamma\right) d}
$$

Here $d$ is the sediment grain size and $\gamma_{s}$ is the specific sediment weight $\gamma_{s}=g \rho_{s}$, where $\rho_{s}$ is the sediment density.

Using (120) and (121), $\tau_{*}$ can be written as a function of the specific gravity or the relative density of fluids $r=\rho_{s} / \rho_{w}$.

$$
\tau_{*}=\frac{g \eta^{2} u^{2}}{(r-1) d R_{h}^{1 / 3}} .
$$

To determine $\tau_{* c}$ many experiments have been performed in different works. Concretely, Shields proposed the well-known Shields-diagram (cf. [40], p. 107).

Some usual formulae for rivers are the following:

- Grass (see [26]) proposed the following formula for the solid transport discharge,

$$
q_{b}=A_{g} u|u|^{m_{g}-1}, 1 \leqslant m_{g} \leqslant 4
$$

where the constant $A_{g}\left(s^{2} / m\right)$ must take into account the grain diameter and the kinematic viscosity. It is usually obtained by experimental data. The usual value of exponent $m_{g}$ is set to $m_{g}=3$.

- Meyer-Peter \& Müller (see [36]) developed one of the most popular formulae for the solid transport discharge,

$$
q_{b}=\sqrt{(r-1) g d^{3}} \operatorname{sgn}(u) 8\left(\tau_{*}-\tau_{* c}\right)^{3 / 2},
$$

where $\tau_{* c}$ usually is set to 0.047 .

- Van Rijn (see [49]) developed the following formula for the solid transport discharge,

$$
q_{b}=\sqrt{(r-1) g d^{3}} \frac{0.005}{C_{D}^{1.7}}\left(\frac{d}{h}\right)^{0.2} \tau_{*}^{1 / 2} \operatorname{sgn}(u)\left(\tau_{*}^{1 / 2}-\tau_{* c}^{1 / 2}\right)^{2.4},
$$

where $C_{D}$ is the drag coefficient.

- Nielsen (see [40]) developed the following formula

$$
q_{b}=\sqrt{(r-1) g d^{3}} \operatorname{sgn}(u) 12 \sqrt{\tau_{*}}\left(\tau_{*}-\tau_{* c}\right) .
$$

In this case the usual value of $\tau_{* c}$ is set equal to $\tau_{* c}=0.05$. 
All these formulae have a range of application which depends on the grain size, the slope of the bottom, the Froude number and the relative density $r$. For example, the M-P\&M formula can be applied if $0.4 \leq d \leq 29 \mathrm{~mm}$, the slope of the bottom is smaller than 0.02 and $1.25 \leq r \leq 4.2$. For more details see [15-17].

Acknowledgements The first author would like to thanks the organizers of the Jacques-Louis Lions Spanish-French school for the invitation. The second author would like to thank the Institute of Mathematics of the University of Seville (IMUS) for the financial support to work on the numerical analysis of models for visco-plastic avalanches.

\section{References}

1. Acary-Robert, C., Fernández-Nieto, E., Narbona-Reina, G., Vigneaux, P.: A well-balanced finite volume-augmented Lagrangian method for an integrated Herschel-Bulkley model. J. Sci. Comput. 53, 608-641 (2012)

2. Ancey, C.: Plasticity and geophysical flows: a review. J. Non-Newtonian Fluid Mech. 142, 4-35 (2007)

3. Ancey, C., Cochard, S.: The dam-break problem for Herschel-Bulkley viscoplastic fluids down steep flumes. J. Non-Newtonian Fluid Mech. 158, 18-35 (2009)

4. Anderson, T.B., Jackson, R.: A fluid mechanical description of fluidized beds. Ind. Eng. Chem. Fundam. 6, 527-539 (1967)

5. Aradian, A., Raphael, E., de Gennes, P.G.: Surface flow of granular materials: a short introduction to some recent models. C. R. Phys. 3, 187-196 (2002)

6. Aranson, I.S., Tsimring, L.S.: Continuum theory of partially fluidized granular flows. Phys. Rev. E. 65, 061303 (2002)

7. Audusse, E., Bristeau, M.-O., Perthame, B., Sainte-Marie, J.: A multilayer Saint-Venant system with mass exchanges for shallow water flows. Derivation and numerical validation. ESAIM Math. Model. Numer. Anal. 45, 169-200 (2011)

8. Balmforth, N.J., Craster, R.V., Rust, A.C., Sassi, R.: Viscoplastic flow over an inclined surface. J. Non-Newtonian Fluid Mech. 139, 103-127 (2006)

9. Batchelor, G.K.: An Introduction to Fluid Dynamics. Cambridge University Press, New Delhi (2000)

10. Bingham, E.C.: Fluidity and Plasticity. Mc Graw-Hill, New York (1922)

11. Bouchut, F., Mangeney-Castelnau, A., Perthame, B., Vilotte, J.P.: A new model of Saint Venant and Savage-Hutter type for gravity driven shallow flows. C. R. Acad. Sci. Paris Ser. I 336, 531536 (2003)

12. Bouchut, F., Fernández-Nieto, E.D., Mangeney, A., Lagree, P.Y.: On new erosion models of Savage-Hutter type for avalanches. Acta Mecha. 199, 181-208 (2008)

13. Bresch, D., Desjardins, B.: Existence of global weak solutions for a 2D viscous shallow water equations and convergence to the quasi-geostrophic model. Commun. Math. Phys. 238, 211223 (2003)

14. Bresch, D., Fernandez-Nieto, E.D., Ionescu, I.R., Vigneaux, P.: Augmented Lagrangian method and compressible visco-plastic flows: Applications to shallow dense avalanches. In: Advances in Mathematical Fluid Mechanics, pp. 57-89. Birkhauser, Basel (2010)

15. Castro-Díaz, M.J., Fernández-Nieto, E.D., Ferreiro, A.: Sediment transport models in Shallow Water equations and numerical approach by high order finite volume methods. Comput. Fluids 37, 299-316 (2008) 
16. Castro-Díaz, M.J., Fernández-Nieto, E.D., Ferreiro, A., Parés, C.: Two-dimensional sediment transport models in shallow water equations: a second order finite volume approach on unstructured meshes. Comput. Meth. App. Mech. Eng. 198, 2520-2538 (2009)

17. Cordier, S., Le, M., Morales de Luna, T.: Bedload transport in shallow water models: why splitting (may) fail, how hyperbolicity (can) help. Adv. Water Resour. 34, 980-989 (2011)

18. Dressler, R.F.: New nonlinear shallow equations with curvature. J. Hydraul. Res. 16, 205-22 (1978)

19. Duvaut, G., Lions, J.-L.: Inequalities in Mechanics and Physics. Springer, Berlin (1976)

20. Einstein, H.A.: The bed load function for sediment transport in open channel flows. Technical Bulletin no. 1026. U.S. Department of Agriculture, Soil Conservation Service, Washington, DC (1950)

21. Fernandez-Nieto, E.D., Bouchut, F., Bresch, D., Castro-Díaz, M.J., Mangeney, A.: A new Savage-Hutter type model for submarine avalanches and generated tsunami. J. Comput. Phys. 227, 7720-7754 (2008)

22. Fernández-Nieto, E.D., Noble, P., Vila, J.P.: Shallow Water equations for Non-Newtonian fluids. J. Non-Newtonian Fluid Mech. 165, 712-732 (2010)

23. Fernández-Nieto, E.D., Koné, E.H., Chacón, T.: A multilayer method for the hydrostatic Navier-Stokes equations: a particular weak solution. J. Sci. Comput. (2013). doi: 10.1007/s10915-013-9802-0

24. Ferrari, S., Saleri, F.: A new two-dimensional shallow water model including pressure effects and slow varying bottom topography. Math. Model. Numer. Anal. 38, 211-234 (2004)

25. Gerbeau, J., Perthame, B.: Derivation of viscous Saint-Venant system for laminar shallow water: numerical validation. Discrete Contin. Dyn. Syst. Ser. B 1, 89-102 (2001)

26. Grass, A.J.: Sediments transport by waves and currents. SERC London Centre for Marine Technology, Report No. FL29 (1981)

27. Gray, J.M.N.T.: Granular flow in partially filled slowly rotating drums. J. Fluid Mech. 441, 1-29 (2001)

28. Heinrich, P., Piatanesi, A., Hébert, H.: Numerical modelling of tsunami generation and propagation from submarine slumps: the 1998 Papua New Guinea event. Geophys. J. Int. 145, 97-11 (2001)

29. Iverson, R.M., Denlinger, R.P.: Flow of variably fluidized granular masses across threedimensional terrain. J. Geophys. Res. 106, 537-552 (2001)

30. Jackson, R.: The Dynamics of Fluidized Particles. Cambridge Monographs on Mechanics. Cambridge University Press, New York (2000)

31. Khakhar, D.V., Orpe, A.V., Andresén, P., Ottino, J.M.: Surface flow of granular materials: model and experiments in heap formation. J. Fluid Mech. 441, 225-264 (2001)

32. Macías, J., Fernández-Salas, L.M., González-Vida, J.M., Vázquez, J.T., Castro Días, M.J., Bárcenas, P., del Río, P., Díaz, V., Morales de Luna, T., de la Asunción, M., Parés, C.: Deslizamientos Submarinos y Tsunamis en el Mar de Alborán. Un ejemplo de modelización, vol. 6. Instituto Español de Oceanografía, Spain (2012)

33. Madsen, P.A., Bingham, H.B., Schaffer, H.A.: Boussinesq-type formulations for fully nonlinear and extremely dispersive water waves: derivation and analysis. Proc. R. Soc. Lond. A Math. Phys. Eng. Sci. 459, 1075-104 (2003)

34. Mangeney-Castelnau, A., Bouchut, F., Vilotte, T.P., Lajeneusse, E., Aubertin, A., Pirulli, M.: On the use of Saint-Venant equations to simulate the spreading of a granular mass. J. Geophys. Res. 110, B09103 (2005)

35. Marche, F.: Theoretical and numerical study of Shallow Water models. Application to Nearshore hydrodynamics. Thesis of the University of Bordeaux, France (2005)

36. Meyer-Peter, E., Müller, R.: Formulas for bed-load transport. Report on 2nd Meeting of International Association for Hydraulic Research, 39-64. Stockholm (2005)

37. Morales de Luna, T.: A Saint Venant model for gravity driven shallow water flows with variable density and compressibility effects. Math. Comput. Model. 47, 436-444 (2008) 
38. Morales de Luna, T., Castro Díaz, M.J., Parés Madroñal, C., Fernández Nieto, E.D.: On a shallow water model for the simulation of turbidity currents. Commun. Comput. Phys. 6(4), 848-882 (2009)

39. Narbona, G., Zabsonre, J., Fernandez-Nieto, E.D., Bresch, D.: Derivation of a bilayer model for shallow water equations with viscosity: numerical validation. Comput. Model. Eng. Sci. 43, 27-71 (2009)

40. Nielsen, P.: Coastal bottom boundary layers and sediment transport. In: Advanced Series on Ocean Engineering, vol. 4. World Scientific Publishing, Singapore (1992)

41. Oswald, P.: Rheophysics: The Deformation and Flow of Matter. Cambridge University Press, New York (2009)

42. Pelanti, M., Bouchut, F., Mangeney, A.: A roe-type scheme for two-phase Shallow granular flows with bottom topography. Math. Model. Numer. Anal. 42, 851-885 (2008)

43. Pirulli, M., Bristeau, M.O., Mangeney, A., Scavia, C.: The effect of the earth pressure coefficients on the runout of granular material. Environ. Model. Softw. 22, 1437-1454 (2007)

44. Pitman, E.B., Le, L.: A two-fluid model for avalanche and debris flows. Philos. Trans. R. Soc. A Math. Phys. Eng. Sci. 363,1573-1601 (2005)

45. Pudasaini, S., Hutter, K.: Avalanche Dynamics. Springer, New York (2007)

46. Saint-Venant, A.J.C.: Théorie du mouvement non-permanent des eaux, avec application aux crues des rivières et à l'introduction des marées dans leur lit. C. R. Acad. Sci. Paris 73, 147-54 (1871)

47. Savage, S.B., Hutter, K.: The dynamics of avalanches of granular materials from initiation to run-out. Acta Mech. 86, 201-223 (1991)

48. Tanner, R.I., Walters, K.: Rheology: An Historical Perspective. Elsevier, Amsterdam (1998)

49. Van Rijn, L.C.: Sediment transport (III): bed forms and alluvial roughness. J. Hydraul. Div. Proc. ASCE 112, 1733-1754 (1984)

50. Wieland, M., Gray, J.M.N.T., Hutter, K.: Channelized free-surface flow of cohesionless granular avalanches in a chute with shallow lateral curvature. J. Fluid Mech. 392, 73-100 (1999) 